九州大学学術情報リポジトリ

Kyushu University Institutional Repository

Palynologisch-stratigraphische Untersuchung der tertiären Schichten im Kasuya und Fukuoka Kohlenfeld von Nordkyushu, Japan

Takahashi, Kiyoshi

Faculty of Sciences, Kyushu University

https://doi.org/10.5109/1524298

出版情報：九州大學理學部紀要：Series D, Geology. 5 (4)，pp.199-221，1957-01-31. Faculty of Science, Kyushu University バージョン：

権利関係 : 


\title{
Palynologisch-stratigraphische Untersuchung der tertiären Schichten im Kasuya und Fukuoka Kohlenfeld von Nordkyushu, Japan
}

\author{
Von
}

\author{
Kiyoshi TAKAHASHI
}

\begin{abstract}
Inhalt
I. Einführung. . . . . . . . . . . . . . . . . . . 199

II. Geologische Fragen . . . . . . . . . . . . . 200

III. Methode der Aufbereitung. . . . . . . . . . . . . . . . 203

IV. Taxonomisches und Nomenklatorisches der Sporomorphae. . . . . . . . 204

V. Stratigraphische Merkmale in den Pollenspektren in beiden Kohlenfeldern 204

a) Kasuya Kohlenfeld . . . . . . . . . . . . . . . . . . . 205

b) Fukuoka Kohlenfeld . . . . . . . . . . . . . . . . 208

VI. Beschreibung der Sporomorphae. . . . . . . . . . . . 215

VII. Zusammenfassung . . . . . . . . . . . . . . . . 219

VIII. Literatur . . . . . . . . . . . . . . . . . . . . 220
\end{abstract}

\section{Einführung}

Seit den zuerst von R. Potonié angestellten systematischen Untersuchungen fossiler Sporen und Pollen haben Fachgenosen weitere stratigraphisch wertvolle Arbeiten geleistet. Besonders sind die neuerdings gemachten auffallenden Fortschritte auf diesem Gebiete $z$ beachten. Man hat schon betont, dass Erforschung der Sporae dispersae nicht nur dem Gebiet der Stratigraphie zustatten kommt, sondern auch zur Klärung der Fragen der reinen Paläobotanik, besonders der Pflanzensoziologie, -ökologie, -geographie, Entwicklungsgeschichte u. a., führen wird. Es wurde ausserdem schon berichtet, dass die genaue eingehende Untersuchung der Sporae dispersae auf dem Gebiete der ökonomischen Geologie, besonders der Kohlengeologie, weiterhin der Erdölgeologie usw., mit grossem Nutzen angewandt werden kann.

In Japan sind leider noch nicht so viele Bearbeitungen der Sporae dispersae in den tertiären und tieferen Sedimenten $z u$ finden. Aber heute beschäftigen sich einige Forscher sehr eifrig mit der Untersuchung fossiler Sporen und Pollen. Auf der Tagung der japanischen geologischen Gesellschaft, April, 1955, wurde die Frage der sog. "Pollenanalyse“ als Thema der Debatte aufgenommen. Um das Problem der Taxonomie und Nomenklatur der Sporomorphae in einer kleinen Abendversammlung 
der japanischen geologischen Tagung, April, 1956, zur Diskussion zu bringen, hat der Verfasser Herrn S. Tokunaga um seine Mithilfe gebeten, aber durch zwingende Umstände wurde das Vorhaben nicht ausgeführt.

Der Verfasser veröffentlicht hier seine Arbeit, die auf dem künstlichen (rein morphographischen) System von P. W. Thomson und H. D. Pflug (1953) beruht. Dieses rein morphographische System ist dem paläontologischen Zweck entsprechend. Nach häufigem und heftigem Meinungsaustausch wurde dieses System und seine pollenstratigraphische Gliederung festgestellt. Solche ausgezeichnete bahnbrechende Arbeiten sind für uns ein starker Antrieb zur Weiterforschung. Herr Dr. H. D. PFLUG hat mir sehr freundlichst seine Forschungsergebnisse betreffs der Taxonomie und Nomenklatur der Sporen und Pollen mitgeteilt. Der Verfasser muss hier Herrn Dr. H. D. Pflug für seine Freundlichkeit von ganzem Herzen danken.

Beim Sammeln der Arbeitsmaterialien ist der Verfasser den Bergmeistern fol gender Kohlenbergwerke zu grossem Dank verpflichtet; Takeuchi-umi, Shin-ôtani, Fukuhaku, Shime, Sawara, Tashima und Fukutoyo Kohlenbergwerke.

Die vorliegende Arbeit ist im geologischen Institut der Universität Kyushu entstanden.

\section{Geologische Fragen}

Der Verfasser hat seine ersten Versuche mit „Pollenanalyse “ an der Steinkohle im Kasuya und Fukuoka Kohlenfeld gemacht, um die Merkmale der Bildungen im japanischen tertiären Pollenspektrum stratigraphisch festzustellen, um dann einige Schlussfolgerungen über die bestehenden Meinungen betreffs der Vergleichung der Schichten aus seiner pollenanalystischen Arbeit zu ziehen. Infolgedessen hat er die Einzelheiten der Pollenspektren einiger Formationen in beiden Feldern klargemacht und die gebräuliche Datierung durch gegenseitigen Vergleich mit ihnen beurteilt. Eine eingehende Darlegung dieser Frage wird im nachfolgenden Kapitel gegeben. Doch hier will der Verfasser die geologischen Fragen in den vorliegenden Bezirken zusammenhängend mit seinen mikropaläontologischen Ergebnissen auseinandersetzen.

Über die geologische Datierung der beiden Kohlenfelder waren die früheren Arbeiten nicht immer gleich. Im letzten Jahre hat R. SAITo gegen die bestehenden Meinungen betreffs der Vergleichung der tertiären Schichten von Nordkyushu bedeutende Bedenken vorgebracht. Die stratigraphischen Einteilungen von H. MATsuSHITA (1949) und R. SAITo (1956) sind folgendermassen (s. Tabelle 1).

Hinsichtlich des Vergleiches der Schichten im Kasuya Kohlenfeld besteht kein grosser Unterschied der Meinungen. H. Matsushita und R. Saito stellen Kasuya Gruppe auf die Nogata-Stufe und bestimmen ihre untere Grenze. Dagegen macht T. NAGAO (1928) sie nicht klar. Bis heute ist kein Fossil ausser Kieselholzresten in diesem Gebiete bekannt. Deswegen ist der zeitliche Vergleich der Schichten mit 
Tabelle 1. Stratigraphische Einteilung im Kasuya und Fukuoka Kohlenfeld [nach Matsushita (1949) \& R. Saito (1956)]

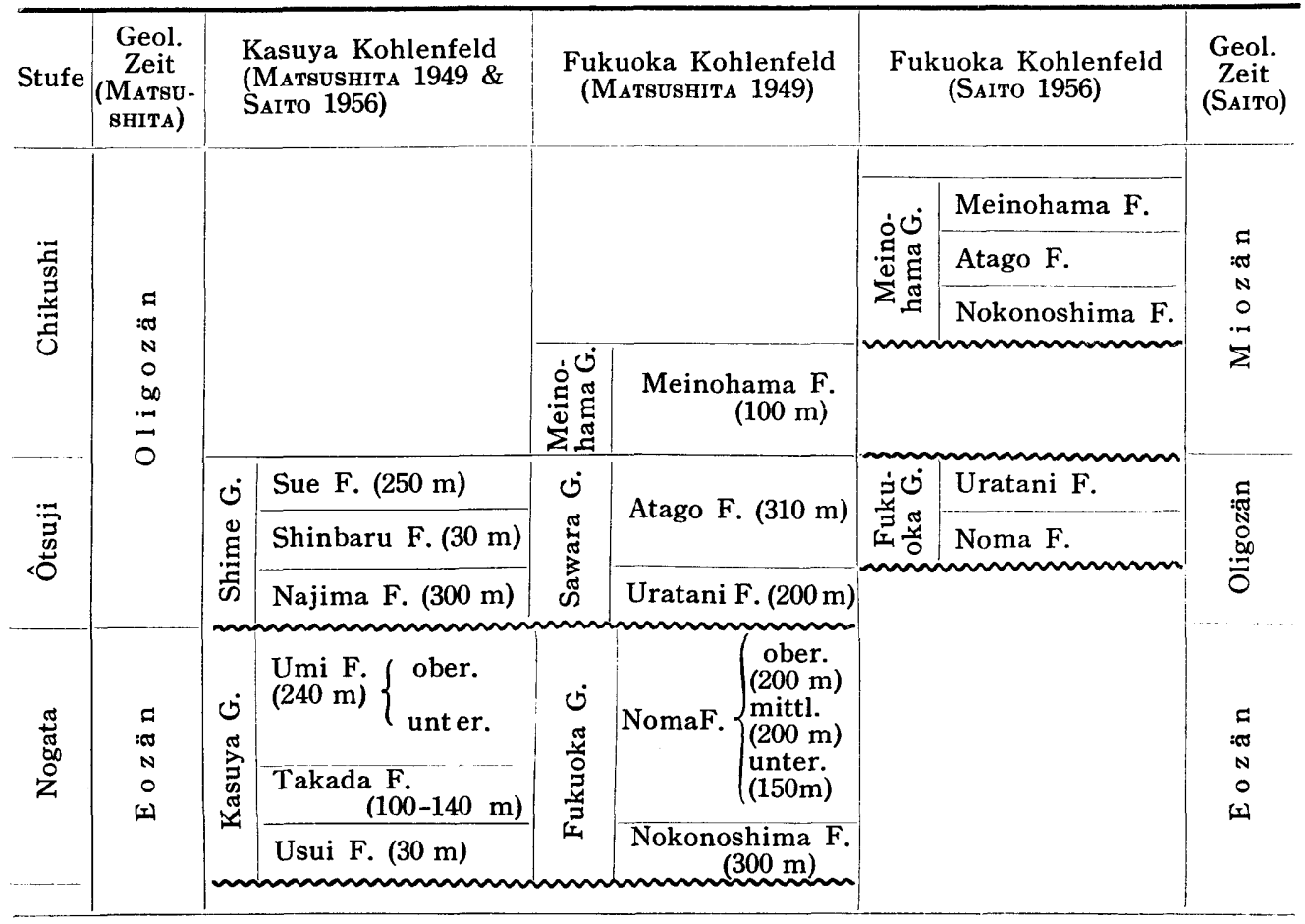

anderen so schwierig, dass man nur Vermutungen darüber anstellen kann. Selbst wenn die Muschelschalereste aus der marinen Najima Formation, die sich auf der Umi Formation diskordanzig aufhäuft, den Vergleich mit den Schichten in anderen Bezirken mit Sicherheit möglich machen, so kann man die untere Grenze der Kasuya Gruppe mit den hergebrachten Kenntnissen doch nicht bestimmen.

Die Muschelschalereste aus der marinen Najima Formation sind von einigen Forschern als Orthaulax japonica NAGAO, Turritella karatsuensis NAGAO, Cultellus izumoensis YоKоYAMA und Ostrea sp. bezeichnet worden und auch aus dem Hangenden des "Mie-Flözes“ der Shinbaru Formation Cyrena? sp. und Unio sp. berichtet.

Die bisher bekannten Makroreste der Pflanzen aus der Kasuya Gegend sind sehr selten. In den Kohlenflözen selbst und ihren Hangenden oder Liegenden kann man manche Kieselhölzer, die entweder liegen oder auf der Ablagerungsfläche aufrecht, Wurzeln schlagend stehen, finden. S. ENDO (1954) hat einige aufrecht stehende Kieselhölzer 1 bis zu $3 \mathrm{~m}$ Durchmesser in der oberen Umi Formation, $1.5 \mathrm{~km}$ Nordwest von Sasaguri, Kasuya, Fukuoka Provinz, als Taxodioxylon sequoianum Gothan vorgefunden. Ausser diesem hat man bis heute kein Fossil gefunden.

Beim Fukuoka Kohlenfeld ist die Frage schwieriger, d. h. es gibt hier zwei verschiedene Meinungen. Auf der einen Seite wird behauptet, dass die Formationen 
im Meinohama Gebiete stratigraphisch in die Chikushi-Stufe gehören. Auf der anderen Seite ist man der Ansicht, dass nur die Meinohama Sandstein Gruppe der ChikushiStufe angehört.

R. SAITo hat das geologische System in diesem Kohlenfeld in zwei Gruppen, d. h. in Fukuoka und Meinohama Gruppe, geteilt. Bei ersterer Gruppe hat er die Noma Formation mit der Shinbaru Formation und die Uratani Formation mit der Sue Formation verglichen und bei letzterer Gruppe hat er die Nokonoshima, Atago und Meinohama Formation als eine Gruppe aufgefasst. Er hat auch eine grosse zeitliche Lücke zwischen beiden Gruppen in Betracht gezogen.

H. Matsushita hat das System in drei Gruppen geteilt. Er hat die Sawara Gruppe mit der Shime Gruppe und die Fukuoka Gruppe mit der Kasuya Gruppe verglichen. Die von ihm definierte Fukuoka Gruppe unterscheidet sich von der Fukuoka Gruppe R. SAITo's. Die Grundursachen solcher Meinungsverschiedenheit liegen darin, dass die Schichten durch grosse Verwerfung und ferner durch die Aufhäufung der Alluvien voneinander entfernt verbreitet sind, und zu allem Unglück kein Molluskenrest in der Nokonoshima, Noma und Uratani Formation bekannt ist.

Die von T. Nagao, H. Matsushita u. a. schon berichteten Molluskenreste aus der Meinohama Formation sind Venercardia subnipponica NAGAO, Callista hanzawai NAGAO, Pitar kyushuensis NagaO, Aturia yokoyamai NagaO, Crassatellites yabei NAGAO usw. und die Reste aus der Atago Formation sind Venercardia subnipponica NAGAO, Callista hanzawai NAGAO, Pitar kyushuensis NGGaO, Cultellus sp. und Epitonium submaculosum NagaO.

In einer Dissertation der Universität Kyushu hat T. Watanabe (1950) hauptsächlich die alttertiären Blattabdrücke aus dem Fukuoka Kohlenfeld behandelt. Infolge der ungenügenden Erhaltungszustände der Fossilien und der Schwierigkeiten, ihre Zugehörigkeit zu bestimmen, wurde keine eingehende Untersuchung durchgeführt. Er hat folgende Makroreste berichtet: Sequoia sp., Quercus sp. und Zelkova sp. aus der Nokonoshima Formation; Equisetum sp., Sequoia sp., Dryophyllum? sp., Quercus sp., Ficus? sp., Cinnamomum sp., Zelkova sp. u. a. aus der Noma Formation; Equisetum sp., Sequoia sp., Castanea sp., Cinnamomum sp., Zelkova sp., Alnus sp., Acer sp. usw. aus der Uratani Formation; Sabalites* sp., Musophyllum sp., Cinnamomum sp., Acer sp. u. a. aus dem Hangenden des "Nanashaku-Flözes“ der Atago Formation. Solche Blattabdrücke spielen keine bedeutende Rolle beim Studium der stratigraphischen Merkmale der Schichten, aber einige von diesen Resten scheinen wichtig zu sein.

Schliesslich hat bisher noch niemand die Aufhäufung der Noma Formation auf der Nokonoshima Formation und der Atago Formation auf der Uratani Formation stratigraphisch oder paläontologisch nachgewiesen. Um diese Aufgabe zu lösen, muss man jedenfalls eingehende paläontologische Untersuchung machen, weil die tertiären

* T. Watanabe (1950) hat einen unvollständig erhaltenen palmartigen Makrorest einer Pflanze als Sabalites beschrieben. Nach seiner Abbildung zu urteilen, scheint der Rest Trachycarpus zu sein. In Japan ist Trachycarpus-Rest bisher nur aus den miozänen Schichten bekannt. Sabalites ist nur in den paläogenen Schichten in Ostasien bekannt. 
Hügel voneinander vereinzelt entfernt stehen und der Vergleich durch die Fazies der Schichten sehr schwierig ist.

Der Verfasser hat die fossilen Sporen und Pollen, die als einziger Schlüssel für den Vergleich der Schichten angesehen werden, mikroskopisch untersucht. Dadurch glaubt zum Schluss er eine Lösung der oben erwähnten Aufgaben gefunden zu haben. Er schliesst sich der Meinung H. Matsushita's an, dass nämlich die untere Noma Formation mit der Takada Formation gleichzeitig ist und dass die obere Noma Formation mit der oberen Umi Formation verglichen werden kann, mit der teilweisen Verbesserung des stratigraphischen Vergleiches der Schichten im südwestlichen Teil des Fukuoka Kohlenfeldes. Das Pollenspektrum der Atago Formation wurde im Kasuya Kohlenfeld nicht gefunden. Die Einzelheiten der Untersuchung der Proben werden im folgenden Kapitel gegeben.

\section{Methode der Aufbereitung}

Die hier behandelten fossilen Sporen und Pollen wurden alle aus den Kohlen der verschiedenen Horizonte in beiden Kohlenfeldern extrahiert. Die verwitterte und weiche Kohle auf dem Ausgehenden enthält nur eine sehr kleine Anzahl der Sporae dispersae. Daher ist solche Kohle auf dem Ausgehenden für die Analyse ungeeignet. Obwohl die Kohle auf dem Ausgehenden gesammelt wurde, ist die harte Kohle für unseren Zweck brauchbar.

Da es sich hier um Steinkohle handelt, so ist ein Aufschliessen nötig, bei dem das Schulzesche Gemisch (Kaliumchlorat und Salpetersäure, mit nachfolgender Alkalibehandlung) die Hauptrolle spielt. Der Verfasser hat sehr gute Ergebnisse mit dieser Aufbereitungsmethode erzielt.

Für einen Aufschluss wurden immer 2-3 g von 50-100 g grob durch filtriertes Wasser gesäuberter und zerkleinerter Kohle verwandt. Dabei muṣs das betreffende Material mit dem Sieb gereinigt werden; es wurde ein Sieb mit 24 Löcher gebraucht. Das Material wird in dem Becherglas $(500 \mathrm{cc})$ oder der Dreieckflasche ( $300-500 \mathrm{cc})$ mit dem Schulzeschen Gemisch behandelt. Darauf wurde es etwa 20 Stunden kalt gestellt und dann anschliessend noch 5-10 Minuten auf dem Wasserbad erhitzt und dann mit durch Glasfilter filtriertem Wasser gefüllt. Die Entferung des Löslichen geschah durch mehrmaliges Dekantieren.

Der dunkelbraun gefärbte Absatz wurde mit 10-15\% igem Kalihydrat etwa 5 Minuten auf dem Wasserbad erhitzt. Nach der Entferung der schwarzbraun gefärbten Lösung wurde der Absatz mehrmals mit durch Glasfilter filtriertem Wasser gewaschen. Bei der Aufbereitung kann die Zentrifugalmaschine, um Zeit zu sparen, benutzt werden.

Dieses Material wurde für die Herstellung der Präparate mit Glyzerin-Gelee verwandt. 
Untersuchung erfolgte mit Fluorit-Objektiven (Apertur 1,32) hauptsächlich bei $912(114 \times 8)-1668(114 \times 12)$ facher Vergrösserung.

\section{Taxonomisches und Nomenklatorisches der Sporomorphae}

Die neue taxonomische Gliederung der Sporomorphae wurde von P. W. Thомson und H. D. Pflug (1953) ausführlich veröffentlicht. Ihre Taxonomie entwickelt sich auf rein morphologischer Grundlage. Sie stellten folgendes fest: „Im Quartär und im jüngsten Tertiär kann man die Sporomorphae meist ohne weiteres entsprechend der botanischen Zugehörigkeit ihrer Träger in das natürliche System einordnen. Im Mitteltertiär treten bereits Schwierigkeiten auf, im Alttertiär und tieferen Abschnitten der Erdgeschichte ist eine Bestimmung nicht mehr möglich, .............. Ein konsequent durchgeführtes künstliches System ist hier die einzige Ordnungsmöglichkeit.“ Man kann auch dasselbe von den anderen Pflanzenfossilien, z. B. den Blattabdrücken, sagen.

Herr Dr. H. D. Pflug hat mir freundlicherweise seine Ansicht über die Taxonomie und Nomenklatur der Sporomorphae mitgeteilt. Der Verfasser schliesst sich seiner Meinung über die Taxonomie und Nomenklatur der Sporomorphae an.

Wenn man fossile Sporen und Pollen untersucht, kommt es zu Meinungsverschiedenheiten über die Aufgaben der Taxonomie und Nomenklatur. In Japan schliessen sich nur einige Palynologen dem künstlichen System an. Um die Aufgabe der Taxonomie und Nomenklatur der Sporomorphae auf der japanischen geologischen Tagung zu diskutieren, hat der Verfasser sich bei Herrn S. Tokunaga bemüht, aber infolge widriger Umstände kam eine Diskussion nicht zustande.

Das künstliche (rein morphographische) System PfLug's wird als ein notwendiges Ergebnis früherer Untersuchungen gültig angewandt. Um das System noch weiter entwickeln zu können, müssen noch weitere Ergebnisse abgewartet werden.

\section{Stratigraphische Merkmale in den Pollenspektren in beiden Kohlenfeldern}

Die schon von P. W. Thоmson und H. D. Pflug (1953) gegebene pollenstratigraphische Gliederung wurde von ihnen durch weitere eingehende Beobachtungen verfeinert [H. D. Pflug 1956). Diese schöne Gliederung hilft uns sehr bei unseren Untersuchungen. Vergleicht man die Veränderung des Pflanzenreiches, das aus Makroresten besteht, mit den aus den Sporen und Pollen bestehenden Reste in Verbindung mit der Stratigraphie, so sind, wie diese Gliederung zeigt, sehr feine und deutliche Verschiedenheiten festzustellen. Es ist allgemein bekannt, dass die Altersbestimmung durch Blattabdrücke wegen des häufigen Wechsels der Namengebung sehr schwierig ist, aber bei Pollen und Sporen wurde im Ausland festgestellt, dass hier ein Vergleich der Schichten von grossem Nutzen ist.

In beiden Kohlenfeldern hat der Verfasser Pollen und Sporen aus vielen Kohlen- 
flözen der verschiedenen Horizonte untersucht. Die interessanten Ergebnisse dieser Arbeit werden hier mitgeteilt, aber solche palynologische Untersuchungen sind in Japan noch sehr selten. Deshalb ist ein Vergleich der vorliegenden Ergebnisse mit denen aus den gleichalterigen Fundschichten in anderen Bezirken jetzt noch nicht möglich. In Kunft hofft der Verfasser den Bereich der Untersuchung noch mehr zu erweitern und durch die Entwicklung der palynologischen Untersuchung ein eingehendes pollenstratigraphisches Schema feststellen zu können. Die vorliegenden Ergebnisse werden als ein Grund künftiger Arbeit gelten können.

a) Kasuya Kohlenfe $1 \mathrm{~d}$

In dem nord-südlich langgestreckten Kasuya Kohlenfeld sind die Entwicklungszustände der Kohlenflöze im Norden und Süden verschieden. Im Süden sind die Kohlenflöze häufiger als die im Norden. Die die Kohlenflöze führenden Formationen werden von der Najima Formation $(300 \mathrm{~m})$ der Transgression auf die Kasuya Gruppe in zwei Gruppen geteilt, d. h. Unterflöz- und Oberflözgruppe. Der Verfasser hat hauptsächlich die pollenführenden Proben aus dem südlichen Gebiete untersucht.

\section{1) Die Unterflözgruppe}

Diese gehört wahrscheinlich ins obere Eozän. Gleichalterige Fundschichten, die zum Vergleich dienen können, werden wahrscheinlich leicht in einigen anderen Bezirken, z. B. in Chikuho, Karatsu u. a., gefunden.

Vergleicht man die Pollenspektren von Kohlenflözgruppe der Takada Formation und von der der Umi Formation, so scheinen sie bei einer oberflächlichen Durchsicht ähnlich zu sein, aber praktisch sind sie im Detail deutlich verschieden. Beide Pollenbilder haben etwa $80 \%$ gemeinsame Formen. Das Pollenspektrum dieser Unterfözgruppe ist in Betreff des Form-Genus dem des obereozänen Borkener Bildes Mitteleuropas ähnlich.

Über die Merkmale der Helmstedter Ober- (mittleres Eozän) und Unterflözgruppe (unteres Eozän) gibt H. D. Pflug (1952) folgendes Bild: „, Es kann also zusammengefasst werden: Innerhalb der Windblütler des extrapalustren Hochwaldes geht im ältesten Tertiär oder der höchsten Kreide die Vorherrschaft vom extratriporaten auf den tricolpaten [cupuliferoiden] Pollen über. Dieser Umbruch liegt unterhalb der Bildungsperiode der Helmstedter Unterflözgruppe. In der Abteilung „Extratriporatae " fassen wir eine Reihe Dreieckpollen enger morphogenetischer Verwandtschaft zusammen, deren Reichtum im Senon ein Maximum erreicht und im Alttertiär langsam ausklingt. "..

In den Sporomorphae aus unserer Unterfözgruppe sind extratriporaten Pollen überhaupt nicht vorhanden. Falls die oben erwähnte mitteleuropäische Veränderung des Pollenbildes auch in den tieferen Schichten als diese Unterflözgruppe in Japan beobachtet wird, so wird das von grossem Interesse sein. Nur eine zukünftige 


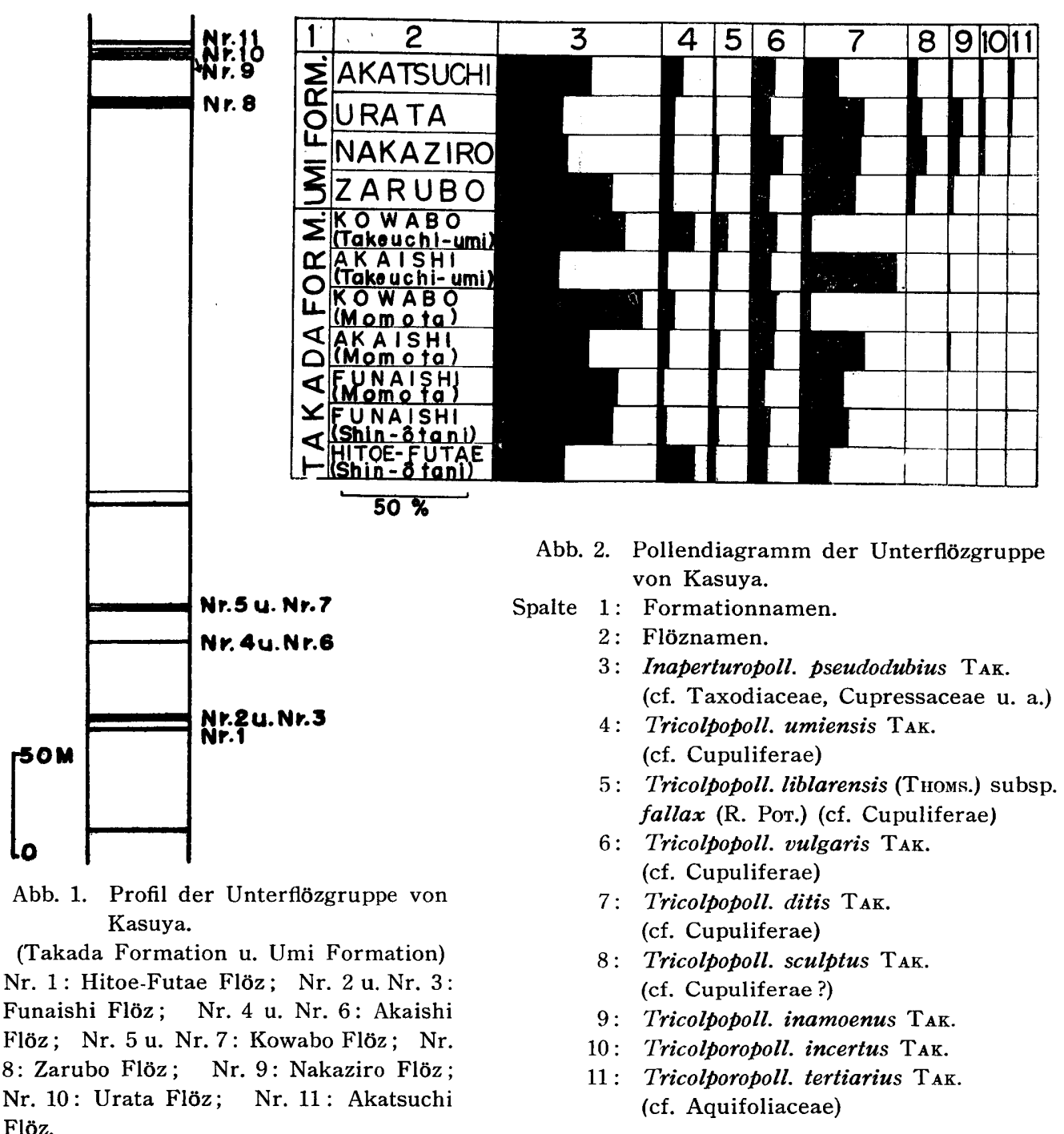

Untersuchung kann über solche Dinge Aufschluss geben. Jedenfalls kann man sich leicht vorstellen, dass das Pollenspektrum der Unterflözgruppe einen Schnitt der vom alten nach dem jüngeren Alter wechselnden Pflanzenwelt zeigen wird. Mann kann nun eine kleine Veränderung in diesem Pollenspektrum erkennen.

In diesem sind praktisch nur zwei vorherrschende Typen, der Inaperturopoll. pseudodubius-Typus in erster Linie und untergeordnet die Tricolpopoll. (wahrscheinlich Cupuliferen)-Gruppe. Der Pollen vom Inaperturopoll. pseudodubius Typus wird wahrscheinlich mit dem Kieselholzrest Taxodioxylon sequoianum Gothan in enger Beziehung stehen. Diese Pflanzen sind die vorherrschenden Windblütler des Bruchwaldes im Niederland, der vom umgebenden näheren Randgebiet des Moores oder vom Moorgebiet selbst aus seinen Pollen weit ausstreut, und zwar in grossen Megen 
sowohl im Verlaufe der Unterflözbildung wie in dem der Oberflözbildung. Die cf. cupuliferoiden Pflanzen des Tricolpopoll.-Typus sind die herrschenden Windblütler des extrapalustren Hochwaldes, der vom erhöhten Randsockel des Moores seinen Pollen weit ins Moorbecken hineinstreut.

Zwischen den Pollenbildern der Takada und Umi Formation wird keine merkliche Veränderung erkannt, aber in der Umi Formation treten die wenigprozentigen neuartigen Pollen, Tricolpopoll. sculptus TAK., Tricolporopoll. tertiarius TAK., Tricolporopoll. incertus. TAK. u. a., auf.

Können solche neue Pollenformen, die im Fukuoka Kohlenfeld ihren hohen Wert gezeigt haben, sich als wichtige Zeitmarken erweisen? Die quantitativen Anteilwerte in den einzelnen Kohlenflözen sind allerdings nicht ohne weiteres vergleichbar. Hier handelt es sich um Vorhandensein oder Nichtvorhandensein der qualitativen Elemente. Ob die oben erwähnten Pollen als Zeitmarken gelten können, werden künftige Untersuchungen vieler gleichalterigen, jüngeren und tieferen Fundschichten bestimmen.

Der Pollen Tricolpopoll. liblarensis (Tномs.) subsp. fallax (R. PoT.), der eine aus dem mitteleuropäischen tiefsten Tertiär berichtete Art ist, tritt hier als die einzige mit Mitteleuropa gemeinsame Spezies auf und zwar in geringen Mengen.

Der geflügelte Pinaceen-Pollen, Pityosporites, zeigt eine ganz geringe Frequenz. Die Sporen treten auch so gering auf, dass man ihre feste Diagnose nicht leicht bestimmen kann. Solche Sporen sind cf. Stereisporites, Concavisporites, Punctatosporites, Cingulatisporites, Divisisporites u. a. Davon tritt cf. Stereisporites häufiger als andere auf.

\section{2) Die Oberflözgruppe}

Da der Verfasser keine Materialien aus der Sue Formation sammeln konnte, kann er leider hier über ihre Sporomorphae nichts berichten. Er will aber das Pollenspektrum der Shinbaru Formation klarmachen. Alle Materialien der Shinbaru Formation wurden im Shime Kohlenbergwerk gesammelt.

Auch hier sind zwei Typen, die Inaperturopoll.-Gruppe und die Tricolpopoll. (cf. Cupuliferen)-Gruppe, herrschend. Unsere Aufmerksamkeit muss dem häufigeren Auftreten gan $z$ anderer Elemente des extrapalustren Hochwaldes geschenkt werden. Die dreieckigen und polyeckigen Pollen treten in grösseren Prozenten als bei der Unterflözgruppe auf. Der dreieckige Triatriopoll.-Pollen, der vielleicht dem MyricaceenPollen gehört, ist neuartig. Solche Erscheinungen der neuen Mitglieder zeigen, dass sich während des Übergangs von der Unterflözgruppe nach der Oberflözgruppe die kleine Vegetationsänderung unter den Hauptwindblütern des extrapalustren Hochwaldes vollzogen hat. Zwei Pollen, Tricolpopoll. sculptus TAK. und Tricolporopoll. tertiarius TAK., die in der Umi Formation als wichtig behandelt wurden, treten noch in dem „, Itsue-Flöz “ mit geringeren Prozenten auf, aber nicht schon in dem „NioiishiFlöz“. Eine Spore, Laevigatosporites dehiscens TAK., erscheint mit geringen Fre- 


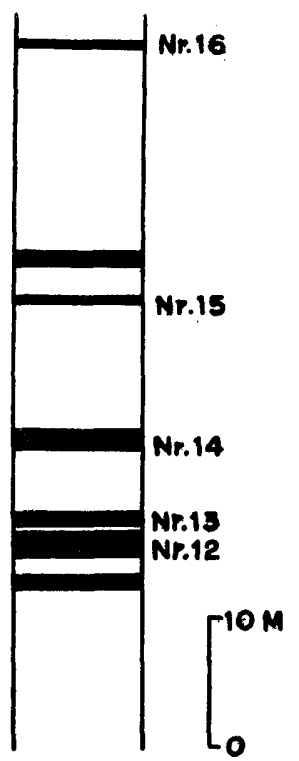

Abb. 3. Profil der unteren Ober. flözgruppe von Kasuya. (Shinbaru Formation)

Nr. 12: Itsue Flöz

Nr. 13: Nioiishi Flöz

Nr. 14: Mie Flöz

Nr. 15: Hitoe Flöz

Nr. 16: Sunaishi Flöz

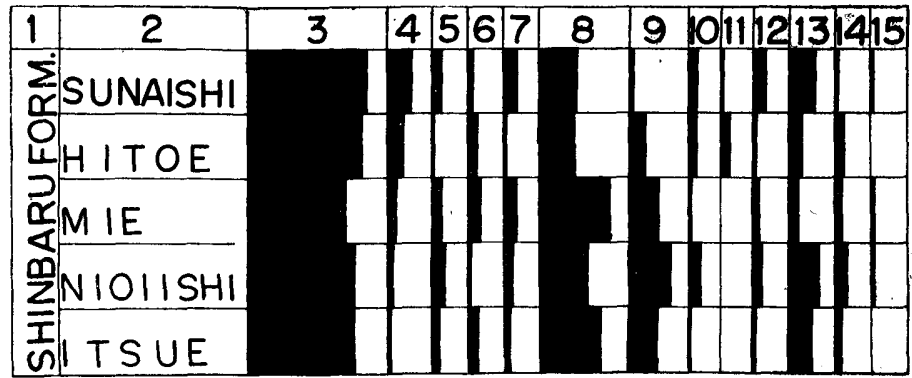

$50 \%$

Abb. 4. Pollendiagramm der Oberflözgruppe von Kasuya.

Spalte 1: Formationnamen

2: Flöznamen

3: Inaperturopoll. pseudodubius TAK.

(cf. Taxodiaceae, Cupressaceae u. a.)

4: Inaperturopoll. laevigatus $\mathrm{T}_{\mathrm{AK}}$.

(cf. Taxodiaceae, Cupressaceae u. a.)

5: Tricolpopoll. umiensis TAK. (cf. Cupuliferae)

6: Tricolpopoll. liblarensis (THомs.) subsp. fallax (R. Pot.) (cf. Cupuliferae)

7: Tricolpopoll. vulgaris TAK. (cf. Cupeliferae)

8: Tricolpopoll. ditis TaK. (cf. Cupliferae)

9: Triporopoll.-Gruppe (Trip. shimensis, Trip. constatus u. Trip. festatus)

10: Subtriporopoll. kyushuensis 'ТАК.

11: Subtriporopoll. levius TAK.

12: Triatriopoll mirabilis TAK. (cf. Myricaceae).

13: Polyvestibulopoll. eminens T $\mathrm{T}_{\mathrm{K}}$. (cf. Alnus)

14: Polyporopoll. grandis TAK. (cf. Ulmaceae)

15: Laevigatosporites dehiscens $\mathrm{T}_{\mathrm{AK}}$. (cf. Polypodiaceae)

quenzen vereinzelt. Inaperturopoll. laevigatus TAK. scheint morphogenetisch mit Inaperturopoll. pseudodubius TAK. der Bruchwaldvegetation in enger Beziehung zu stehen. Der herrschende Pollen von Tricolpopoll.-Gruppe ist Tricolpopoll ditis TAK., wie bei der Unterflözgruppe.

b) Fukuoka Kohle nfeld

Der Hauptteil des Fukuoka Kohlenfeldes liegt im Westen und Südwesten von Fukuoka. In diesen Bezirken sind einige Aufgaben über den Vergleich der Schichten noch vorhanden.

Im Westen hat der Verfasser einige Materialien der Kohlen der Atago Formation im Sawara Kohlenbergwerk gesammelt. Im Südwesten wurden die Materialien in Fukutoyo und Tashima Kohlenbergwerken gesammelt.

Die alttertiären Schichten des Sitzortes des Fukutoyo Kohlenbergwerks wurden nach Untersuchung H. Matsushita's als obere Noma Formation mit der oberen Umi Formation verglichen und auch die Schichten des Sitzortes von Tashima Kohlenbergwerk wurden von ihm in die obere Noma Formation eingeordnet. Seine Vergleiche wurden hauptsächlich durch die Schichtenfazies festgestellt, da kein Fossil 
bekannt ist. Im letzten Jahre hat R. SAITo die Noma Formation mit der Shinbaru Formation des Kasuya Kohlenfeldes verglichen. Der Verfasser hat die Pollenspektren strittiger Schichten klargemacht und ist dabei $\mathrm{zu}$ folgendem Schluss gekommen. Das Pollenspektrum der Schichten in der Nähe des Fukutoyo Kohlenbergwerkes ist deutlich dem der Takada Formation des Kasuya Kohlenfeldes ähnlich, und das Pollenspektrum der Shichten in der Umgegend des Tashima Kohlenbergwerkes kann un$z$ weifelhaft mit dem der oberen Umi Formation von Kasuya identifiziert werden.

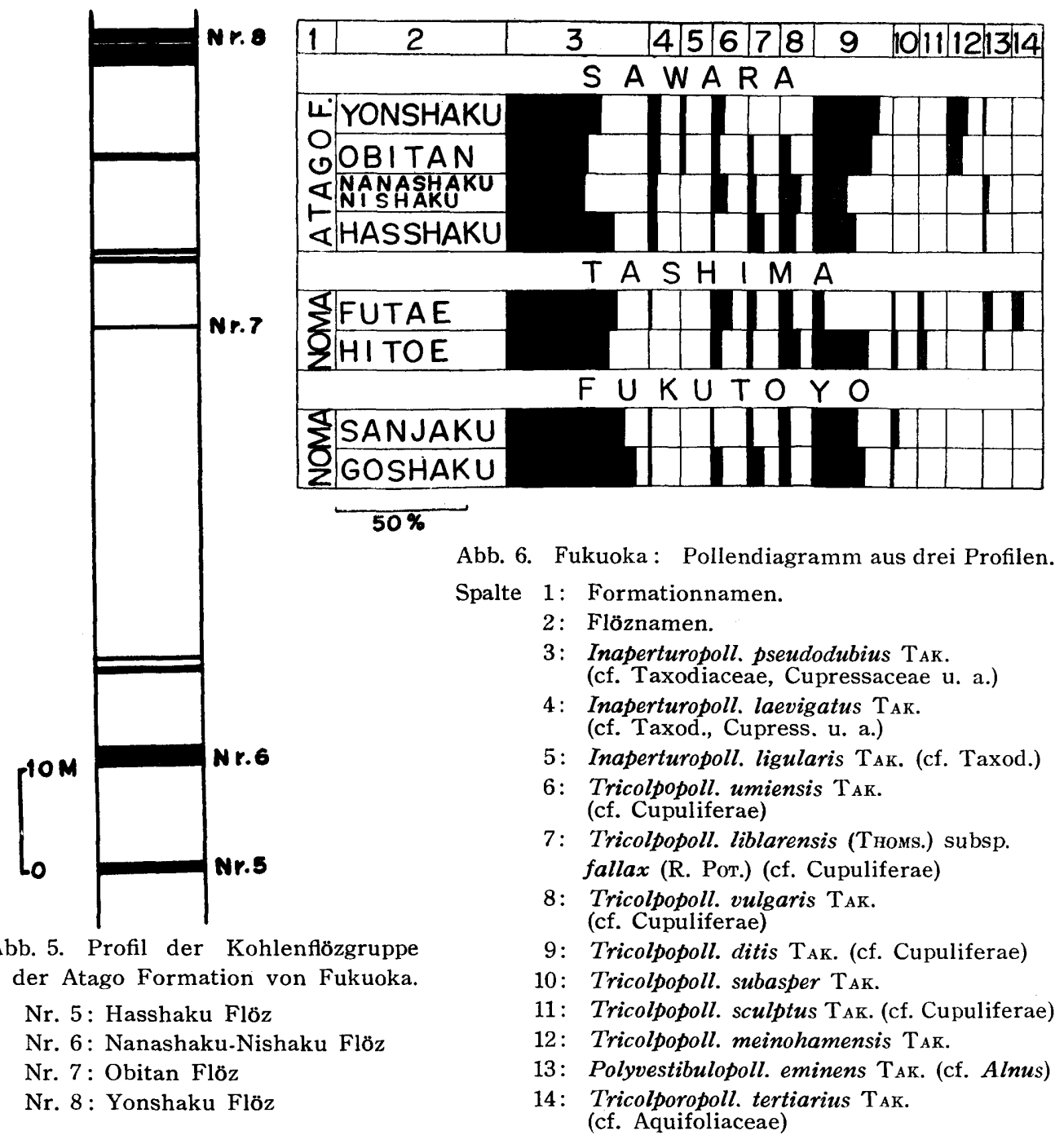

Das Pollendiagramm von Fukuoka Kohlenfeld ist folgendermassen: Das Pollenspektrum aus den im Fukutoyo Kohlenbergwerk gesammelten Proben ist dem der Takada Kohlenflözgruppe ähnlich. In ihm sind hauptsächlich herrschend zwei Typen, die Inaperturopoll.-Gruppe in erster Linie und untergeordnet die Tricolpopoll.-Gruppe. 
Die Zustände der damaligen Vegetation sind hier verhältnismässig deutlich zu erkennen.

Ebenso wie das Pollenbild der Umi Formation von Kasuya, zeigt das Pollenspektrum aus dem Tashima Kohlenbergwerk die Eintritte einiger neuartigen Pollen in die Pflanzenwelt mit kleineren Frequenzen. Die neuen Mitglieder sind Tricolpopoll. sculptus TAK., Tricolporopoll. tertiarius TAK. u. a. Dieser kleine Umbruch ist hier wichtig. Diese Schichten von Tashima, die obere Noma Formation, sind deshalb mit der oberen Umi Formation von Kasuya gleichalterig.

Das Pollenbild der Atago Formation ist von den schon vom Verfasser untersuchten Pollenbilden von Kasuya verschieden. Die unteren und die oberen Atago Formationen werden im Detail der Pollenbilder sich unterschieden. In der oberen Atago Formation treten einige Neupollen auf, Inaperturopoll.ligularis TAK., Tricolpopoll. meinohamensis TAK., usw. Es kann also festgestellt werden, dass sich während der Bildungsperiode der Atago Kohlenflöze eine kleinere Vegetationsänderung vollzogen hat.

H. Matsushita hat die obere Atago Formation mit der Sue Formation von Kasuya verglichen und die untere Atago Formation mit der Shinbaru Formation. R. SAITo stellt, wie schon oben erwähnt, die Atago Formation als eine Gruppe mit der Nokonoshima Formation und der Meinohama Formation auf der Chikushi-Stufe.

Der Verfasser konnte leider kein Pollenbild der Sue Formation untersuchen, aber das Pollenspektrum der Shinbaru Formation wird mit dem der unteren Atago Formation nicht verglichen.

Jedenfalls zeigt das Pollenspektrum der Atago Formation neue Merkmale. Die Datierung der Atago Formation wird erst nach künftigen Untersuchungen der anderen Bezirken bestimmt werden können.

Tabelle 2. Pollenzähltabelle aus den Ober- und Unterflözen des Kasuya Kohlenfeldes.

\begin{tabular}{|c|c|c|c|c|c|c|c|c|c|c|c|c|c|c|c|c|}
\hline Formation-Namen & & Tal & cada & For & rmat & ion & & & & ni & & & $\begin{array}{l}\text { Sh } \\
\text { For }\end{array}$ & & & \\
\hline $\begin{array}{c}\text { Proben-Nr. } \\
\text { Pollenarten }\end{array}$ & 1 & 2 & $\mathbf{3}$ & 4 & 5 & 6 & 7 & 8 & 9 & 10 & 11 & 12 & 13 & 14 & $\mathbf{1 5}$ & 16 \\
\hline $\begin{array}{cc}\text { Laevigatospor. dehiscens } & \mathrm{T}_{\mathrm{AK}} . \\
\text { (cf. Polypodiaceae) } & \text { (MS) }\end{array}$ & & & & & & & & & & & + & & 1 & 1 & & 1 \\
\hline $\begin{array}{l}\text { Monocolpopoll. universalis TAK. } \\
\text { (cf. Palmae) }\end{array}$ & 8 & 4 & 2 & 8 & 4 & 9 & + & 5 & 5 & + & 3 & 1 & & 2 & 8 & 1 \\
\hline Monocolpopoll. verrucatus $\mathrm{T}_{\mathrm{AK}}$ (MS) & & & & & 1 & + & + & & & & + & & & & & \\
\hline $\begin{array}{l}\text { Inaperturopoll. pseudodubius } \mathrm{T}_{\mathrm{AK}} \\
\text { (cf. Taxodiac., Cupressac. u. a.) }\end{array}$ & 28 & 47 & 49 & 37 & 58 & 25 & 51 & 46 & 28 & 26 & 37 & 34 & 34 & 31 & 36 & 38 \\
\hline $\begin{array}{l}\text { Inaperturopoll. laevigatus } \mathrm{T}_{\mathrm{AK} .} \\
\quad \text { (cf. Taxodiac., Cupressac. u. a.) }\end{array}$ & & 1 & & & & 3 & 2 & & . & 1 & 1 & 1 & 2 & 3 & 5 & 8 \\
\hline Inaperturopoll. immutatus $\mathrm{T}_{\mathrm{AK} .}$ & & + & + & + & & + & & + & & + & & & & & & \\
\hline $\begin{array}{l}\text { Triatriopoll. mirabilis TAK. } \\
\text { (cf. Myricaceae) }\end{array}$ & & & & & & & & & & & & 1 & 3 & 2 & 1 & 4 \\
\hline
\end{tabular}




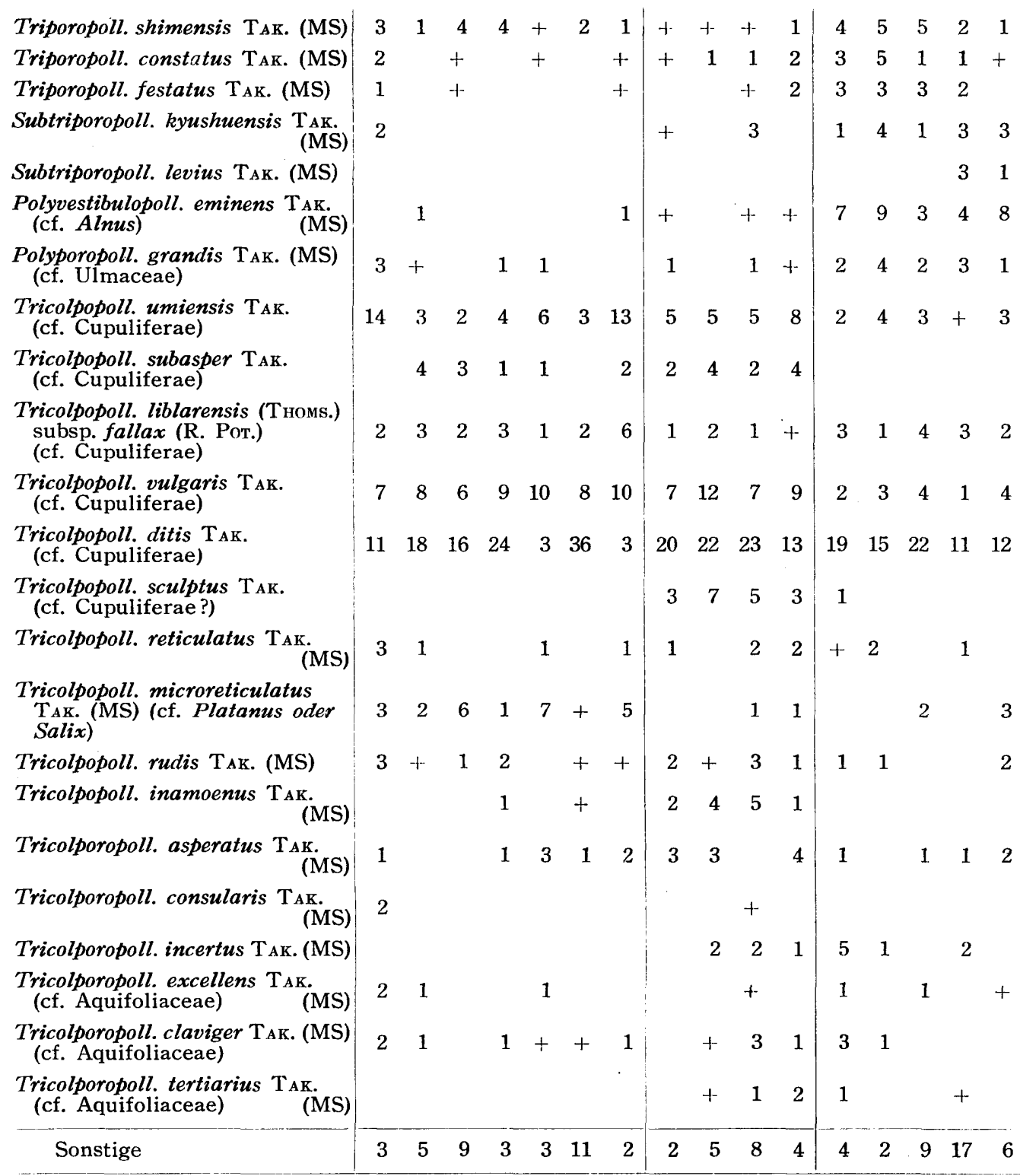

Proben-Nr. 1: Hitoe-Futae Flöz (Shin-ôtani)

2: Funaishi Flöz (Shin-otani)

3: Funaishi Flöz (Momota)

4: Akaishi Flöz (Momota)

5: Kowabo Flöz (Momota)

6: Akaishi Flöz (Takeuchi-umi)

7: Kowabo Flöz (Takeuchi-umi)

8: Zarubo Flöz
9: Nakaziro Flöz
10: Urata Flöz
11: Akatsuchi Flöz
12: Itsue Flöz
13: Nioiishi Flöz
14: Mie Flöz
15: Hitoe Flöz
16: Sunaishi Flöz

+ : Diese Bezeichnung zeigt das Vorhandensein des Pollen, der bei der Zählung nicht aufgetreten ist. 
Wenn man sich einen Überblick über die Pollenspektren der Kohlenflöze in beiden Kohlenfeldern verschafft, so kann man leicht erkennen, dass sich während der Bildungsperiode der Flöze keine merkliche Vegetationsänderung unter den Hauptwindblütlern des Bruchwaldes oder des extrapalustren Hochwaldes vollzogen hat. Die Spektren sind relativ uniform, aber die kleineren Veränderungen der Vegetation sind sehr zu beachten, ganz abgesehen davon, ob solche Veränderungen für das Vergleichen der Tertiärschichten von Wert sind oder nicht. Es ist schon von anderen Forschern festgestellt werden, dass die geringere Zahl der windblütigen Arten mehr eine Eigentümlichkeit subtropischer bis tropischer Breiten, als der kühleren Klimazonen ist. In den Kohlenmooren von Kasuya und Fukuoka sind nur einige windblütige Pollengruppen, von denen jede mehrere botanische Arten umfassen kann,

Tabelle 3. Pollenzähltabelle aus einigen Kohlenflözen im Fukuoka Kohlenfeld.

\begin{tabular}{|c|c|c|c|c|c|c|c|c|}
\hline Formation-Namen & $\begin{array}{l}\text { Nor } \\
\text { (Fuk }\end{array}$ & $\begin{array}{l}\text { F. } \\
\text { oyo) }\end{array}$ & $\begin{array}{l}\text { Nor } \\
\text { (Tas }\end{array}$ & ma) & & go $F$ & $\mathrm{rmat}$ & \\
\hline $\begin{array}{l}\text { Proben-Nr. } \\
\text { Pollenarten }\end{array}$ & $\mathbf{1}$ & 2 & 3 & 4 & 5 & 6 & 7 & 8 \\
\hline $\begin{array}{l}\text { Laevigatospor. dehiscens } \mathrm{T}_{\mathrm{AK}} \text { (MS) } \\
\text { (cf. Polypodiaceae) }\end{array}$ & & & & 1 & & & & + \\
\hline $\begin{array}{l}\text { Monocolpopoll. universalis } \mathrm{T}_{\mathrm{AK}} \\
\text { (cf. Palmae) }\end{array}$ & 4 & 4 & 4 & $\dashv$ & 6 & 2 & 7 & 2 \\
\hline Monocolpopoll. verrucatus $\mathrm{T}_{\Delta \mathrm{K} .}$ (MS) & 1 & & & & & & & \\
\hline $\begin{array}{l}\text { Inaperturopoll. pseudodubius } \mathrm{T}_{\mathrm{AK}} \\
\text { (cf. Taxodiac., Cupressac. u. a.) }\end{array}$ & 50 & 46 & 40 & 43 & 42 & 31 & 32 & 37 \\
\hline $\begin{array}{l}\text { Inaperturopoll. laevigatus TAK. } \\
\text { (cf. Taxodiac., Cupressac. u. a.) }\end{array}$ & 1 & 1 & & 1 & 3 & 3 & 4 & 4 \\
\hline Inaperturopoll. ligularis $\mathrm{T}_{\mathrm{AK}}$ (MS) & & & & & & & 2 & 2 \\
\hline Inaperturopoll. crassatus $\mathrm{T}_{\mathrm{AK}}$ (MS) & & & & & & 1 & 1 & 4 \\
\hline Inaperturopoll. immutatus ТАK. (MS) & & 2 & & & & & & \\
\hline Triporopoll. shimensis ТАK. (MS) & 1 & 2 & 2 & 5 & 2 & 5 & 3 & \\
\hline Triporopoll. constatus $\mathrm{T}_{\mathrm{AK}}$ (MS) & + & & & 2 & & + & & 1 \\
\hline Triporopoll. festatus $\mathrm{T}_{\mathrm{AK}}$ (MS) & 1 & 2 & 1 & 1 & & 3 & 1 & 1 \\
\hline Subtriporopoll. kyushuensis TАK. (MS) & & & 1 & 1 & & + & 1 & \\
\hline $\begin{array}{l}\text { Polyvestibulopoll. eminens } \mathrm{T}_{\mathrm{AK}} \text { (MS) } \\
\text { (cf. Alnus) }\end{array}$ & & & + & 3 & 1 & 2 & & 1 \\
\hline $\begin{array}{l}\text { Polyporopoll. grandis Tak. (MS) } \\
\text { (cf. Ulmaceae) }\end{array}$ & & + & 2 & 1 & 1 & & 1 & 1 \\
\hline $\begin{array}{l}\text { Tricolpopoll. umiensis } \mathrm{T}_{\mathrm{AK}} \\
\quad \text { (cf. Cupuliferae) }\end{array}$ & 4 & 1 & 4 & 8 & 1 & 6 & 3 & 5 \\
\hline $\begin{array}{l}\text { Tricolpopoll. subasper ТАK. } \\
\text { (cf. Cupuliferae) }\end{array}$ & 1 & 3 & 2 & 1 & & & & \\
\hline $\begin{array}{l}\text { Tricolporopoll. liblarensis (Tномs.) } \\
\text { subsp. fallax (R. Рот. (cf. Cupuliferae) }\end{array}$ & 6 & 2 & 2 & 3 & 6 & 3 & 2 & \\
\hline $\begin{array}{l}\text { Tricolpopoll. vulgaris } \mathrm{T}_{\text {AK. }} \\
\text { (cf. Cupliferae) }\end{array}$ & 4 & 4 & 8 & 5 & 6 & 8 & 4 & \\
\hline $\begin{array}{l}\text { Tricolpopoll. ditis } \mathrm{T}_{\Lambda \mathrm{K}} \\
\quad \text { (cf. Cupuliferae) }\end{array}$ & 20 & 17 & 21 & 4 & 16 & 13 & 22 & 25 \\
\hline Tricolpopoll. meinohamensis $\mathrm{T}_{\mathrm{AK}}$ (MS) & & & & & & & 6 & 8 \\
\hline Tricolpopoll. facetus $\mathrm{T}_{\mathrm{AK}}$ (MS) & & & & & 2 & 2 & & \\
\hline
\end{tabular}


Tricolpopoll. sculptus TAK. (cf. Cupuliferae?)

Tricolpopoll. reticulatus $\mathrm{TAK}_{\mathrm{K}}$ (MS)

Tricolpopoll. microreticulatus $\mathrm{T}_{\mathrm{AK}}$. (MS) (cf. Platanus oder Salix)

Tricolpopoll. rudis $\mathrm{TAK}_{\mathrm{AK}}$ (MS)

Tricolpopoll. inamoenus $\mathrm{T}_{\mathrm{AK}}$ (MS)

Tricolporopoll. asperatus $\mathrm{T}_{\mathrm{AK}}$. (MS)

Tricolporopoll. incertus TAK. (MS)

Tricolporopoll. excellens TAK. (MS)

(cf. Aquifoliaceae)

Tricolporopoll. claviger TAK. (MS)

(cf. Aquifoliaceae)

Tricolporopoll. tertiarius $\mathrm{T}_{\mathrm{AK}}$. (MS)

(cf. Aquifoliaceae)

Sonstige

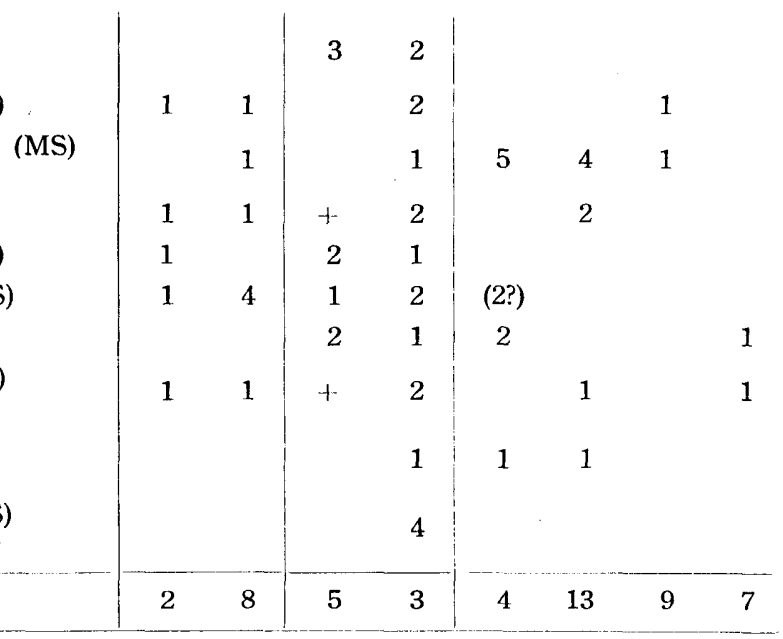

Proben-Nr. 1: Goshaku Flöz

4: Futae Flöz

2: Sanjaku Flöz

5: Hasshaku Flöz

8: Yonshaku Flöz
3: Hitoe Flöz

6: Nanashaku-Nishaku Flöz

7: Obitan Flöz

+ : Diese Bezeichnung zeigt das Vorhandensein des Pollen, der bei der Zählung nicht aufgetreten ist.

massgebend am Pollenspektrum beteiligt, ihre Summe beträgt etwa 80 bis $90 \%$ der Pollensepktren.

Die Ziffer selbst auf den vorgebrachten Pollenzahlentabellen zeigt das wahre Verhältnis der damaligen Pflanzen nicht immer richtig, denn der grosse Unterschied zwischen den Wind- und Insektenblütlern liegt in der Pollenproduktivität und der Pollenstreuungsweise, und ausserdem gibt es viele Pollen, die als Fossil nicht erhalten bleiben und verschwinden. Es handelt sich hier nicht um die Ziffer selbst, sondern um das Auftreten oder Nichtauftreten beschränkter Pollenarten und ferner um die relativen Pollenfrequenzen. Untersucht man die Sporae dispersae in den Sedimentgesteinen, so muss man natürlich nach der Veränderung der wenigen Pflanzenarten forschen. Die Pflanzen mit den als Fossil nicht erhaltbaren Pollen, z. B. das Krautgewächs, kommen deshalb als Gegenstand unserer Untersuchung nicht in Frage. Nach den Arten der Pollen und Sporen führenden Sedimentsgesteine, z. B. Sandstein, Ton, Kohle, u. a., können die Pollenspektren aus ihnen jedes für sich besondere Eigentümlichkeiten aufweisen, da z. B. die Pollenspektren aus den marinen Gesteinen die von weither transportierten Hochwaldpollen in grosser Menge zeigen, während die aus den Kohlenfözen mehr die Elemente des Bruchwaldes aufzeigen. Es wird für die Gebiete der Paläobotanik, besonders der Pflanzensoziologie, -geographie, -ökologie und Entwicklungsgeschichte, und auch der Stratigraphie von grosser Bedeutung sein, auf diese Weise von der Gestalt der vergangenen Pflanzenwelt ein klares Bild $z \mathrm{u}$ bekommen.

Wie H. D. Pflug (1952) schon geschrieben hat, muss man als Zeitmarken zuerst „Leitfossilien“ feststellen und dazu noch „Leitfrequenzen“. 
Der Verfasser will hier noch einiges Wichtige erwähnen.

Tricolpopoll. umiensis TAK., Tricolpopoll. liblarensis (Tномs.) subsp. fallax (R. Poт.), Tricolpopoll. vulgaris TAK., Tricolpopoll. ditis TAK. u. a., cf. Cupuliferae, bilden die wichtigen windblütigen Gruppen des extrapalustren Hochwaldes. Diese Gruppe bestreitet durchschnittlich etwa ein Halb bis ein Drittel der Hundertsätze. Tricolpopoll. liblarensis (Thoms.) subsp. fallax (R. Рот.) ist eine mitteleuropäische tertiäre Pollenform. Tricolpopoll. sculptus TAK. und Tricolpopoll. subasper TAK. sind feinstratigraphisch wichtig. Tricolpopoll. sculptus TAK. tritt nur in der oberen Unterflözgruppe von Kasuya und Fukuoka und auch im untersten Kohlenflöz (Itsue-Flöz) der Oberflözgruppe von Kasuya auf. Tricolpopoll subasper TAK. tritt nur in der Unterflözgruppe von Kasuya auf.

Tricolporopoll.-Gruppe ist im Durchschnittspektrum nur in einem geringen Prozentsatz vertreten. Tricolporopoll. asperatus TAK., Tricolporopoll. incertus. TAK. und Tricolporopoll. tertiarius TAK. werden feinstratigraphisch wichtige Pollenformen sein. Da Tricolporopoll.-Gruppe hier gering auftritt, so werden ihre Einzelheiten in den nachfolgenden Arbeiten klargemacht.

Dreieckpollen (+Polyeckpollen): In der Unterflözgruppe von Kasuya sind sie etwa unter $10 \%$ des Pollenspektrums. Im Pollenspektrum der Oberflözgruppe von Kasuya nehmen sie dagegen durchschnittlich etwa $20 \%(+)$ des Spektrums ein. Im Fukuoka Kohlenfeld sind 10\% (-) des Durchschnittspektrums Dreieckpollen. Im Oberfözspektrum ist die Zunahme der Häufigkeit der Dreieckpollen (+Polyeckpollen) merkwürdig und mag vielleicht wichtig sein. Triatriopoll. mirabilis TAK. ist hier besonders wichtig. Das neue Auftreten von Triatriopoll. mirabilis TAK. ist ein wichtiges Merkmal im Oberflözspektrum. Über die Dreieckpollen wird der Verfasser in einer besonderen Arbeit eingehend berichten.

Die Monocolpopoll.-Gruppe macht nicht ganz 10\% des Durchschnittspektrums aus. Monocolpopoll. universalis TAK. (cf. Palmae) spielt hier die Hauptrolle, aber seine Veränderung der Auftretenfrequenz ist kaum festzustellen. Dieser Pollen gehört vielleicht zur Palmae. Im Kohlenfeld von Nordkyushu wurden die Makroreste Sabalites nipponicus (KRYsht.) aus den Schichten der "Ôtsuji-Stufe " bisher oft vorgefunden. Die zeitlich beschränkte Uppigkeit von Sabalites ist einfach durch das Urteil der vorliegenden Pollenspektren vielmehr schwarzsehend. Wenn die Konvergenzerscheinung der Palmae-Pollen vorhanden ist, so sind die Verhältnisse anders. Diese Aufgabe muss noch weiterhin untersucht werden.

Ungeffügelter Koniferen-Pollen Inaperturopoll. pseudodubius TAK. (cf. Taxodiaceae, Cupressaceae u. a.) reicht im Durchschnitt nahe an $40 \%$ heran. In beiden Kohlenfeldern werden viele Kieselhölzer vom Taxodiaceen-Typus gefunden. Inaperturopoll. pseudodubius TAK. scheint mit diesem Holz, Taxodioxylon sequoianum Gothan, eine enge Verbindung $\mathrm{zu}$ haben. Inaperturopoll. ligularis TAK. tritt nur im Atago- 
Pollenspektrum auf. Geflugelter Koniferen-Pollen Pityosporites tritt nur vereinzelt auf.

Die Vorgenannten sind die hauptwindblütigen Gruppen.

Die Insektenblütler sind zwar formenarm. Tricolporopoll.-Gruppe vom Aquifoliaceen-Typus erregt hier unsere Aufmerksamkeit. Tricolporopoll. tertiarius TAK. ist stratigraphisch verhältnismässig wichtig.

Einige Sporen treten nur vereinzelt auf. Laevigatosporites (Polypodiaceen-Spore) zeigt ein Auftreten von nur etwa $1 \%$, doch das ist stratigraphisch wichtig.

\section{Beschreibung der Sporomorphae}

Der Verfasser konnte viele Pollenformen aus den tertiären Kohlenflözen unterscheiden, aber die Pollen und Sporen, die vereinzelt auftreten, werden hier noch nicht beschrieben, weil ihre Zahl gering ist und die Bestimmung ihrer Grösse, ihrer Exinendicke u. a. noch nicht genug erforscht ist. Daher werden hier nur einige beobachtete Pollen beschrieben.

Oberabteilung: Pollenites R. POT.

I. Abteilung: Bilateres Pflug

Formgattung; Monocolpopollenites Pflug \& Thомson

Monocolpopollenites universalis $\mathrm{n}$. sp.

Tafel 38, Fig. 6-9; Tafel 39, Fig. 11.

Typus: s. Tafel 38, Fig. 9; Präparat GK·V 165-2.

Diagnose: 15-36 $\mu$. Exine bis $1 \pm \mu$ dick, stets chagrenat oder intrapunktat, seltener intrarugulat. Der Äquator hat eine charakteristische asymmetrische Kontur, mit etwas abgerundeten Ecken. Eine Ecke liegt meist an dem Schnittpunkt mit der Colpus-Ebene. Der Colpus liegt nicht symmetrisch. Colpus klafft selten. Figura linsenförmig.

Typus : $32.6 \times 20.5 \mu$. Exine $0.9 \mu$ dick. Ektexine und Endexine etwa gleichstark. Struktur intrapunktat. Der Äquator hat eine asymmetrische Kontur, mit abgerundeten Ecken. Colpuswülste $1 \mu$ breit.

Bemerkungen: Diese Art ist der mitteleuropäischen ältesten Tertiärart Monocolpopoll. zievelensis PF. und der alttertiären Art Monocolpopoll. tranquillus (R. Pот.) sehr ähnlich. Die vorliegende Art kann aber nach Exinenstruktur von Monocolpopoll. zievelensis PF. und nach Grösse, Exinenstärke und Äquator-Kontur von Monocolpopoll. tranquillus (R. РОт.) unterschieden werden.

Botanische Zugehörigkeit: Wahrscheinlich Palmae.

Fundpunkte: Shin-ôtani, Momota, Takeuchi-umi, Fukuhaku und Shime von Kasuya; Fukutoyo, Tashima und Sawara von Fukuoka. Typus; Urata-Flöz der oberen Umi Formation, Fukuhaku Kohlenbergwerk, Kasuya. 


\section{Abteilung: I n a pertures Pflug \& Thomson \\ Formgattung: Inaperturopollenites PfLug \& TномsоN \\ Sektion: M a g n o i d a e Pflug \& Thomson \\ Inaperturopollenites pseudodubius $\mathrm{n}$. sp.}

Tafel 38, Fig. 11-17; Tafel 39, Fig. 13-14.

Typus: s. Tafel 38, Fig. 17 ; Präparat GK-V 255.

Diagnose: $15-64 \mu ; 20-40 \mu$ am häufigsten vorkommend. Figura ursprunglich kugelig, meist stark verfaltet, aber oft durch spez. Quellung aufgeplatzt, hierdurch eine charakteristische schnabelartige Öffnung aufweisend (Hiatus). Exine scheinbar einschichtig (Apertur 1,32), sehr dünn, seltener bis $0.5 \mu$ dick, chagrenat bis intrapunktat, zuweilen schwach rugulat.

Typus: $33 \mu$. Figura kugelig, stark verfaltet. Exine sehr dünnwandig (einschichtig), intrapunktat.

Bemerkungen: Eine europäische Art Inaperturopoll. dubius (R. POT. \& VEN.) ist der vorliegenden Art Inaperturopoll. pseudodubius TAK. sehr ähnlich. Die letztere kann nach Grösse, Exinenstärke u. a. von der ersteren klar unterschieden werden.

Botanische Zugehörigkeit: In erster Linie kommen Taxodiaceen-Cupressineen in Frage; denn, beobachtet man rezente Pollen von Taxodiaceen oder Cupressineen, so kann man in vielen Fällen ähnliche Formen erhalten.

Fundpunkte: Shin-ôtani, Momota, Takeuchi-umi, Fukuhaku und Shime von Kasuya; Fukutoyo, Tashima und Sawara von Fukuoka. Typus: Funaishi-Flöz der Takada Formation, Momota Kohlenbergwerk, Kasuya.

\section{Inaperturopollenites laevigatus $\mathrm{n}$. $\mathrm{sp}$.}

Tafel 38, Fig. 18; Tafel 39, Fig. 16.

Typus: s. Tafel 39, Fig. 16; Präparat GK-V 542.

Diagnose : 18-50 . Figura kugelig, sekundär verfaltet, sehr selten durch spez. Quellung aufgeplatzt, hierdurch eine charakteristische schnabelartige Öffnung aufweisend (Hiatus). Exine scheinbar einschichtig (Apertur 1,32), fast sehr dünnwandig, aber sehr selten bis $0.8 \mu$ dick. Exine immer glatt.

Typus: $38 \mu$. Figura kugelig, stark verfaltet. Exine sehr dünnwandig (einschichtig). Exine glatt.

Bemerkungen: Die vorliegende Art ist den europäischen Pollenformen Inaperturopoll. dubius (R. РоT. \& VEN.) und Inaperturopoll. hiatus (R. POT.) ähnlich, aber sie kann hauptsächlich nach Skulptur von Inaperturopoll. dubius (R. PoT. \& VEN.) und nach Exinenstärke und -struktur von Inaperturopoll. hiatus (R. Pot.) unterschieden werden.

Botanische Zugehörigkeit: Vielleicht Taxodiaceae, Cupressaceae u. a.

Fundpunkte: Sin-ôtani, Takeuchi-umi, Fukuhaku und Shime von Kasuya; Fuku- 
toyo, Tashima und Sawara von Fukuoka. Typus: Yonshaku-Flöz der Atago Formation, Sawara Koblenbergwerk von Fukuoka.

III. Abteilung; Lo n g a o n es Pflug

Formgattung: Tricolpopollenites Pflug \& Thomson

Sektion: As peroida e Pflug

Tricolpopollenites umiensis $\mathrm{n}$. sp.

Tafel 38, Fig. 37-39; Tafel 39, Fig. 31-32.

Typus: s. Tafel 38, Fig. 39 ; Präparat GK-V 330.

Diagnose: 18-37 $\mu$ Figura spindelförmig. Breitenlängenindex $z$ wischen 0.4 und 0.8. Ektexine und Endexine etwa gleichstark, an der Polregion ist die Exine stärker. Exine 0.4 bis $1.3 \mu$ dick. Struktur intrabaculat oder intrarugulat. Colpen selten Geniculus. Polhemisphäre meist zugespitzt, aber auch halbkugelig.

Typus: $33.5 \times 21 \mu$. Figura spindelförmig. Breitenlängenindex ca. 0.6. Ektexine und Endexine etwa gleichstark. Exine $1 \mu$ dick an seinem Äquator, aber an der Polregion etwas stärker. Struktur intrabaculat. Colpen fast parallel verlaufend. Polhemisphäre etwas zugespitzt.

Bemerkungen: Die vorliegende Spezies ist den mitteleuropäischen tertiären Arten Tricolpopoll henrici (R. Pot.), Tricolpopoll. microhenrici (R. Pot.) oder Tricolpopoll. pudicus (R. PoT.) ähnlich. Sie kann nach Grösse, Exinenstärke und Breitenlängenindex von Tricolpopoll. henrici (R. Рот.) und Tricolpopoll. pudicus (R. Рот.) und nach Struktur und Breitenlängenindex von Tricolpopoll. microhenrici (R. Рот.) unterschieden werden.

Botanische Zugehörigkeit: Dürfte von Cupuliferen aus der Verwandtschaft der Gattung Quercus stammen.

Fundpunkte: Shin-ôtani, Momota, Takeuchi-umi, Fukuhaku und Shime von Kasuya; Fukutoyo, Tashima und Sawara von Fukuoka. Typus: Akatsuchi-Flöz der oberen Umi Formation, Shime Kohlenbergwerk, Kasuya.

Tricolpopollenites subasper n. sp.

Tafel 38, Fig. 42-43.

Typus: s. Tafel 38, Fig. 42; Präparat GK-V 303.

Diagnose : $14-25 \mu$. Figura kugelig oder nach der Polachse gestreckt. Breitenlängenindex ca. 0.8 bis 1 . Exine chagrenat, unter $1 \mu$ dick (häufigst 0.6 bis $0.8 \mu$ dick). Colpen selten Geniculus. Polhemisphäre halbkugelig oder unterhalbkugelig.

Typus : $23 \times 18.4 \mu$. Figura etwas kugelig. Breitenlängenindex 0.8. Exine chagrenat, $0.6 \mu$ dick. Polhemisphäre halbkugelig.

Bemerkungen: Die hier bestehende Art Tricolpopoll. subasper TAK. ist der mitteleuropäischen tertiären Form Tricolpopoll. asper Pflug \& THomson ähnlich, aber die erstere wird hiermit als selbständige Spezies betrachtet, da sie hauptsächlich nach Grösse von der letzteren unterschieden werden kann. 
Stratigraphisches Verhalten: Nur aus der Unterflözgruppe von Kasuya und den Kohlenflözen der Noma Formation von Fukuoka bekannt.

Botanische Zugehörigkeit: Vielleicht Cupuliferae.

Fundpunkte: Shin-ôtani, Momota, Takeuchi-umi, Fukuhaku und Shime von Kasuya; Fukutoyo und Tashima von Fukuoka. Typus: Nakaziro-Flöz der oberen Umi Formation, Fukuhaku Kohlenbergwerk, Kasuya.

\section{Tricolpopollenites liblarensis (Tномs.) subsp. fallax (R. PoT.)}

Tafel 38, Fig. 40-41; Tafel 39, Fig. 35-37, 59b.

Diagnose: s. P. W. Thomson und H. D. Pflug (1953), S. 96-97.

Fundpunkte: Shin-ôtani, Momota, Takeuchi-umi, Fukuhaku und Shime von Kasuya ; Fukutoyo, Tashima und Sawara von Fukuoka.

\section{Tricolpopollenites vulgaris $\mathrm{n}$. $\mathrm{sp}$.}

Tafel 38, Fig. 44-45; Tafel 39, Fig. 38.

Typus: s. Tafel 38, Fig. 45; Präparat GK-V 163.

Diagnose: $14-36 \mu$. Figura spindelförmig bis ellipsoidisch. Breitenlängenindex 0.4 bis 0.8 . Exine schwach chagrenat oder chagrenat. Ektexine und Endexine etwa gleichstark. Exine unter $1 \mu$ dick. Polhemisphäre zugespitzt.

Typus : $25 \times 17.2 \mu$. Figura ellipsoidisch. Breitenlängenindex ca. 0.7. Exine schwach chagrenat. Ektexine und Endexine etwa gleichstark. Exine $0.8 \mu$ dick. Polhemisphäre zugespitzt.

Bemerkungen: Die vorliegende Form wird als selbständige Spezies betrachtet, da sie sich in Breitenlängenindex und Polkappenkontur von Tricolpopoll. subasper TAK. unterscheidet.

Botanische Zugehörigkeit: Vielleicht Cupuliferen.

Fundpunkte: Shin-ôtani, Momota, Takeuchi-umi, Fukuhaku und Shime von Kasuya; Fukutoyo, Tashima und Sawara von Fukuoka. Typus: Urata-Flöz der oberen Umi Formation, Fukuhaku Kohlenbergwerk, Kasuya.

Tricolpopollenites ditis n. sp.

Tafel 38, Fig. 46-49; Tafel 39, Fig. 39-44.

Typus: s. Tafel 38, Fig. 49 ; Präparat GK-V 165-1.

Diagnose: $15-47 \mu$. Figura spindelförmig bis ellipsoidisch. Breitenlängenindex 0.4 bis 0.8 . Exine chagrenat oder intrapunktat, oft etwas rugulat. Polhemisphäre meist zugespitzt. Exine sehr eng vereinigt, oft an der Polregion sehr stark.

Typus: $31.1 \times 19.2 \mu$. Figura spindelförmig. Breitenlängenindex ca. 0.6. Exine intrapunktat. Polhemisphäre etwas zugespitzt. Exine sehr eng vereinigt, aber an der Polregion $1.2 \mu$ dick. 
Bemerkungen: Dieser Pollen ist dem mitteleuropäischen Tricolpopoll. henrici(oder microhenrici-) Typus oder dem japanischen Tricolpopoll. umiensis-Typus ähnlich, aber er kann nach Exinenstärke und Struktur von ihnen unterschieden werden.

Botanische Zugehörigkeit: Vielleicht Cupuliferen.

Fundpunkte: Shin-ôtani, Momota, Takeuchi-umi, Fukuhaku und Shime von Kasuya; Fukutoyo, Tashima und Sawara von Fukuoka. Typus: Urata-Flöz der oberen Umi Formation, Fukuhaku Kohlenbergwerk, Kasuya.

Tricolpopollenites sculptus n. sp.

Tafel 38, Fig. 50-51; Tafel 39, Fig. 33-34.

Typus: s. Tafel 38, Fig. 51; Präparat GK-V 155.

Diagnose : $20-37 \mu$. Figura spindelförmig oder ellipsoidisch. Breitenlängenindex zwischen ca. 0.5 und 0.8. Exine ca. $1 \mu \pm$ dick $(0.7-1.5 \mu$ dick). Ektexine etwa doppelt so dick wie Endexine. Ektexine unregelmässig schwach vertieft, d. h. schwach corrugatartig. Seitenlinien an Langachsern schwach konvex. Polhemisphäre etwas zugespitzt, aber selten halbkugelig oder unterhalbkugelig.

Typus: $32.1 \times 20 \mu$. Figura spindelförmig. Breitenlängenindex ca. 0.6. Exine $1.2 \mu$ dick, an der Polregion etwas stärker und schwach intrabaculatartig. Ektexine etwa doppelt so dick wie Endexine. Ektexine unregelmässig corrugatartig vertieft. Polkappenkontur zugespitzt.

Bemerkungen: Diese ist der miteinander auftretenden Art Tricolpopoll umiensis TAK. ähnlich, aber sie wird als selständige Spezies betrachtet, da sie sich in Exinenskulptur oder -struktur und im Verhältnis der Ektexine- und Endexinestärke von Tricolpopoll. umiensis TAK. unterscheidet.

Botanische Zugehörigkeit; Vielleicht Cupuliferen (?).

Fundpunkte: Fukuhaku und Shime von Kasuya; Tashima von Fukuoka. Typus: Urata-Flöz der oberen Umi Formation, Fukuhaku Kohlenbergwerk, Kasuya.

\section{Zusammenfassung}

Die Kohlenlager von Kasuya und Fukuoka wurden palynologisch untersucht und auch das Pollenspektrum jeder Kohlenflözgruppe wurde erläutert.

Zwischen Unterflözgruppe und Oberflözgruppe des Kasuya Kohlenfeldes, die durch die marinen Schichten (Najima Formation) getrennt sind, bestehen palynologische Unterschiede. Und auch zwischen den unteren und oberen Schichten in der Unterflözgruppe selbst kann man kleine, aber wichtige palynologische Unterschiede erkennen.

Im Fukuoka Kohlenfeld wurden die Kohlenlager der Noma Formation und der Atago Formation palynologisch untersucht. Es wurde versucht, die palynologischstratigraphischen Merkmale des Fukuoka Kohlenfeldes mit denen der Kohlenflözgruppe von Kasuya zu vergleichen. Das Pollenspektrum der oberen Noma Formation, die 
in der Umgegend am Tashima Kohlenbergwerk liegt, ist dem der oberen Unterflözgruppe ähnlich. Das Pollenspektrum der Noma Formation in der Umgegend des Fukutoyo Kohlenbergwerkes ist mit dem der unteren Unterfözgruppe (Takada Formation) identisch. Im Kasuya Kohlenfeld ist ein dem Pollenspektrum der Atago Formation ähnliches Spektrum noch nicht gefunden worden.

Am Ende der Abhandlung wurden einige Pollenformen beschrieben.

Man kann erkennen, dass die Untersuchung der Sporae dispersae für die Erkenntnis der Veränderungsgeschichte des Pflanzenreiches und für die Erforschung der stratigraphischen Verhältnisse von grosser Bedeutung ist.

\section{Literatur}

Bradiey, W. H. (1931): Origin and Microfossils of the oil shale of the Green River Formation of Colorado and Utah. U.S. Geol. Surv. Prof. Paper, 168, 1-158.

Enpo, S. (1954): Das Klima der Umi-Zeit im Kasuya Kohlenfeld von Kyushu. (Jap.), Jour. Geol. Soc. Japan, 60, 706, 307.

ERdtman, G. (1952): Pollen morphology and plant taxonomy, Angiosperms. Stockholm.

Just, T. (1951): Mesozoic plant microfossils and their geological significance. Jour. Paleont., $25,6,729-735$.

KirchHeimer, F. (1950): Mikrofossilien aus Saltzablagerungen des Tertiärs. Palaeontogr., B, 90, 127-160.

Kremp, G. (1949): Pollenanalytische Untersuchung des miozänen Braunkohlenlagers von Konin an der Warthe. Palaeontogr., B, 90.

KuYl, O. S., Muller J. \& W Wterbork, H. T. (1955): The application of palynology to oil geology, with special reference to western Venezuela. Geol. en Mijnbouw, NW. S., 17e Jg., 3, 49-75.

LEsCHIK, G. (1951): Mikrobotanisch-stratigraphische Untersuchung der jungpliozänen Braunkohle von Buchenau (Kr. Hünfeld). Palaeontogr., B, 92, 1-51.

Matsushita, H. (1949): Geology of Coal Fields in Northern Kyushu. (Jap.), Jour. Mining, Kyushu, Spec. edit.

NAGAO T. (1928) : Stratigraphie der alttertiären Schichten von Kyushu. (Jap.), Jour. Geogr., 40 , no. $467-472$.

Norem. W. L. (1953): Separation of spores and pollen from siliceous rocks. Jour. Paleont., 27, $6,881-883$.

(1953): Classification of spores and pollen for paleontologic correlation. Jour. Paleont. 27, 6, 902.

PrLUG, H. D. (1950) : Vorläufige Ergebnisse einer mikropaläontologischen und stratigraphischen Untersuchung im Braunkohlenlager von Helmstedt. Braunkohle, Wärme u. Energ., Heft 19/20, 340-342.

(1952): Palynologie und Stratigraphie der eozänen Braunkohlen von Helmstedt. Paläont. Z., 26, 112-137.

(1953): Zur Entstehung und Entwicklung des angiospermiden Pollens in der Erdgeschichte. Palaeontogr., B, 95, 60-171.

(1956) : Beiträge zur Klimageschichte Islands II. Sporen und Pollen von Tröllatunga (Island) und ihre Stellung zu den pollenstratigraphischen Bildern Mitteleuropas. N. Jb. Geol. Paläont., Abh. 102, 3, 409-430.

Poroní, R. (1950); Stand der mikropaläobotanischen Tertiärstratigraphie. Z. deutsch. geol. Ges., 97, 54-65. 
(1951): Revision stratigraphisch wichtiger Sporomorphen des mitteleuropäischen Tertiärs. Palaeontogr., B, 91, 131-151.

Reissinger, A. (1939): Die „Pollenanalyse“ ausgedehnt auf alle Sedimentgesteine der geologischen Vergangenheit. Palaeontogr., 84, 1-20.

(1950): Die „Pollenanalyse “ ausgedehnt auf alle Sedimentgesteine der geologischen Vergangenheit. Palaeontogr. B, 90, 99-126.

Ross, N. (1949): On a Cretaceous Pollen and Spore bearing clay deposit of Scania. Bull. geol. Inst. Univ. Upsala, 34, 25-43.

SAIto, R. (1956) : Einige Aufgaben der alttertiären Schichten in den Kohlenfeldern von Japan. (Jap.). Foraminifera, 5, Sondernr. (I) 3-11.

Sears, P. B. (1950): Pollen analyses in Old and New Mexico. Bull. geol. Soc. America, 61, 10, 1171.

SEWARD, A. C. (1919): Fossil plants. IV.

Thiergart, F. (1940): Die Mikropaläontologie als Pollenanalyse im Dienst der Braunkohlenforschung. Brennstoff-Geol., 13. (1947): Leitpollen der untermiozänen und oberoligozänen Braunkohle und ihre systematische Stellung. Z. deutsch. geol. Ges., 97, 54-65.

(1953) : Über einige Sporen und Pollen der Perutzer Schichten (Böhmen). Palaeontogr., B, 95, 53-59.

Thomson, P. W. (1949): Alttertiäre Elemente in der Pollenfiora der rheinischen Braunkohle und einige stratigraphisch wichtige Pollenformen derselben. Palaeontogr., B, 90, 94-96. (1952): Die Sukzession der Pflanzenvereine und Moortypen im Hauptflöz der rheinischen Braunkohle mit einer Übersieht über die Vegetationsentwicklung im Tertiär Mitteleuropas. Bericht geobot. Forschungsinst. Rübel in Zürich. 81-87.

(1954): Der Fazieswechsel im Hauptflöz der rheinischen Braunkohle im Gebiet der Grube Fortuna. Geol. Jb., 69, 329-338.

Thomson, P. W. \& Pflug, H. D. (1952): Die alttertiäre Braunkohle der Tongrube Zievel im Antweiler Graben bei Saltzvey/Bl. Euskirchen. N. Jb. Geol. Paläont., Abh. 96, 1-26.

(1953): Pollen und Sporen des mitteleuropäischen Tertiärs. Palaeontogr., B, 94, 1-138.

Tokunaga S. (1955): Pollenanalyses of the Known Coalseams in the Bibai Area, Sorachi District, Ishikari Coal Field, Hokkaido. (Jap., mit engl. Resumé) M. Rep. Jap. Geol. Surv., $6: 9,11-32$.

Traverse, A. \& Barahoorn, E. S. (1953): Micropaleontology of the Brandon lignite, an early Tertiary coal in central Vermont. (Preliminary note). Jour. Paleont., 27, 2, 289-293.

Ueno, J. (1952): Morphology of pollen of Metasequoia, Sciadopitys and Taiwania. Jour. Inst. Polyt. Osaka City Univ., D, Biol., 2, 23-26.

WatanaBe, T. (1950) : Palaeogene flora of Fukuoka. (Jap., mit engl. Resumé). Diss. Univ., Kyushu.

Weyland, H. \& Kriegrr, W. (1953): Die Sporen und Pollen der Aachener Kreide und ihre Bedeutung für die Charakterisierung des mittleren Senons. Palaeontogr., B, 95, 6-29.

_..___ \& GReifeld G. (1953): Über strukturbietende Blätter und pflanzliche Mikrofossilien aus den untersenonen Tonen der Gegend von Quedlinburg. Palaeontogr., B, 95, 30-52.

Wodehouse, R. P. (1935) : Pollen Grains.

YaMASAKI, T. (1933): Die Morphologie der Pollen und Sporen. (Jap.). Bericht Versuchspflanz. d. Univ. Kyoto (Enshurin-Hokoku). 5. 
K. TAKAHASHI

Palynologisch-stratigraphische Untersuchung der tertiären Schichten im Kasuya und Fukuoka Kohlenfeld von Nordkyushu, Japan

\section{Tafelerkälrung}




\section{Erklärung der Tafel 38}

Kasuya Kohlenfeld (Unterflöz- und Oberflözgruppe)

(Alle Objekte etwa $600 \times$ )

Fig. 1. Stereisporites? sp. Zarubo Fz. (Fukuhaku Kbw.)

Fig. 2. Stereispor. sp. Funaishi Fz. (Momota Kbw.)

Fig. 3. Divisispor. sp. Akaishi Fz. (Takeuchi-umi Kbw.)

Fig. 4. Tuberculatispor. sp. Urata Fz. (Fukuhaku Kbw.)

Fig. 5. Laevigatospor. dehiscens Takahashi (MS) Itsue Fz. (Shime Kbw.)

Fig. 6-9. Monocolpopollenites universalis TAK. n. sp. Fig. 9: Typus; Fig. 6-9: Urata Fz. (Fukuhaku Kbw.)

Fig. 10. Monocolpopoll. verrucatus TAK. (MS) Kowabo Fz. (Takeuchi-umi Kbw.)

Fig. 11-17. Inaperturopoll. pseudodubius TAK. n. sp. Fig. 17: Typus; Fig. 11, 16, 17 : Funaishi Fz. (Momota Kbw.) ; Fig. 12, 13: Kowabo Fz. (Momota Kbw.) ; Fig. 14, 15 : Urata Fz. (Fukuhaku Kbw.)

Fig. 18. Inaperturopoll. laevigatus $\mathrm{T}_{\mathrm{AK}}$. n. sp. Urata Fz. (Fukuhaku Kbw.)

Fig. 19-20. Inaperturopoll. immutatus TAK. (MS) Fig. 19: Urata Fz. (Fukuhaku Kbw.); Fig. 20: Funaishi Fz. (Shin-ôtani Kbw.)

Fig. 21. Pityosporites sp. Istue Fz. (Shime Kbw.)

Fig. 22. Triatriopoll. mirabilis $\mathrm{T}_{\mathrm{AK}}$. (MS) Itsue Fz. (Shime Kbw.)

Fig. 23-24. Triporopoll. shimensis TAK. (MS) Urata Fz. (Fukuhaku Kbw.)

Fig. 25-29. Triporopoll. constatus TAK. (MS) Fig. 25, 27 : Funaishi Fz. (Momota Kbw.); Fig. 26, 29: Itsue Fz. (Shime Kbw.); Fig. 28: Akatsuchi Fz. (Shime Kbw.)

Fig. 30-31. Triporopoll. festatus $\mathrm{T}_{\mathrm{AK}}$. (MS) Fig. 30: Urata Fz. (Fukuhaku Kbw.); Fig. 31 : Itsue Fz. (Shime Kbw.)

Fig. 32-33. Subtriporopoll. kyushuensis TaK. (MS) Fig. 32: Hitoe Fz. (Shime Kbw.); Fig. 33: Itsue Fz. (Shime Kbw.)

Fig. 34. Subtriporopoll. levius TAK. (MS) Hitoe Fz. (Shime Kbw.)

Fig. 35. Polyvestibulopoll. eminens TAK. (MS) Kowabo Fz. (Takeuchi-umi Kbw.)

Fig. 36. Polyporopoll. grandis $\mathrm{T}_{\mathrm{AK}}$. (MS) Urata Fz. (Fukuhaku Kbw.)

Fig. 37-39. Tricolpopoll. umiensis TAK. n. sp. Fig. 39: Typus; Fig. 37 : Zarubo Fz. (Fukuhaku Kbw.) ; Fig. 38: Urata Fz. (Fukuhaku Kbw.); Fig. 39: Akatsuchi Fz. (Shime Kbw.)

Fig. 40-41. Tricolpopoll. liblarensis (Tномs.) subsp. fallax (R. Рот.) Fig. 40: Kowabo Fz. (Momota Kbw.); Fig. 41: Nakaziro Fz. (Fukuhaku Kbw.)

Fig. 42-43. Tricolpopoll. subasper $\mathrm{T}_{\mathrm{AK}}$. n. sp. Fig. 42: Typus; Nakaziro Fz. (Fukuhaku Kbw.)

Fig. 44-45. Tricolpopoll. vulgaris $\mathrm{T}_{\mathrm{AK}}$. n. sp. Fig. 45: Typus; Fig. 44: Zarubo Fz. (Fukuhaku Kbw.) ; Fig. 45: Urata Fz. (Fukuhaku Kbw.)

Fig. 46-49. Tricolpopoll. ditis TAK. n. sp. Fig. 49: Typus ; Fig. 46, 47, 49: Urata Fz. (Fukuhaku Kbw.); Fig. 48: Zarubo Fz. (Fukuhaku Kbw.)

Fig. 50-51. Tricolpopoll. sculptus TAK. n. sp. Fig. 51: Typus; Fig. 50: Zarubo Fz. (Fukuhaku Kbw.); Fig. 51 : Urata Fz. (Fukuhaku Kbw.)

Fig. 52-53. Tricolpopoll. reticulatus TAK. (MS) Urata Fz. (Fukuhaku Kbw.)

Fig. 54-55. Tricolpopoll. microreticulatus TAK. (MS) Urata Fz. (Fukuhaku Kbw.)

Fig. 56. Tricolpopoll. rudis TAK. (MS) Urata Fz. (Fukuhaku Kbw.)

Fig. 57-58. Tricolpopoll. inamoenus TAK. (MS) Fig. 57: Urata Fz. (Fukuhaku Kbw.); Fig. 58: Hitoe Fz. (Shime Kbw.)

Fig. 59. Tricolporopoll. consularis TAK. (MS) Urata Fz. (Fukuhaku Kbw.)

\section{Anmerkung zu den Tafeln}

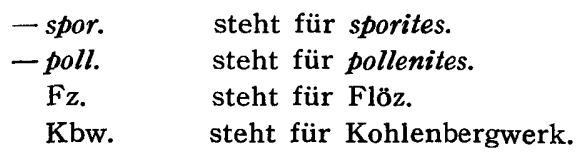



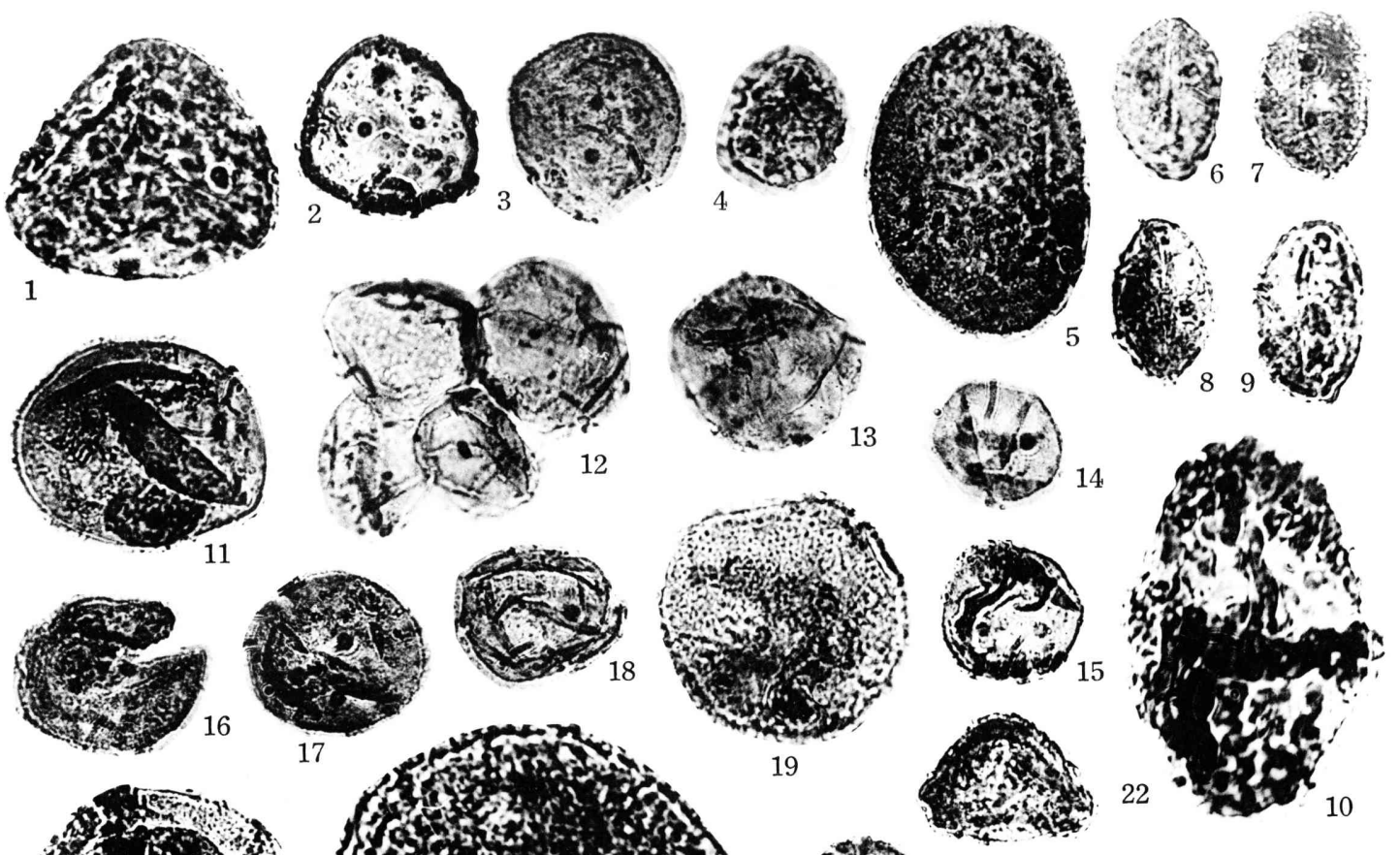

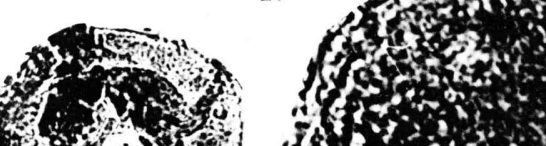

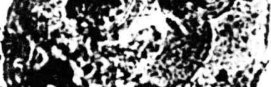

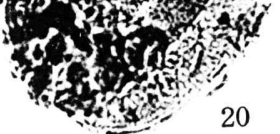

If 10 .

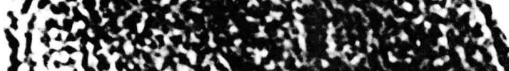

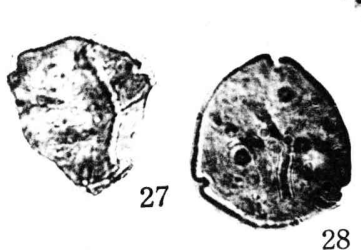
fits
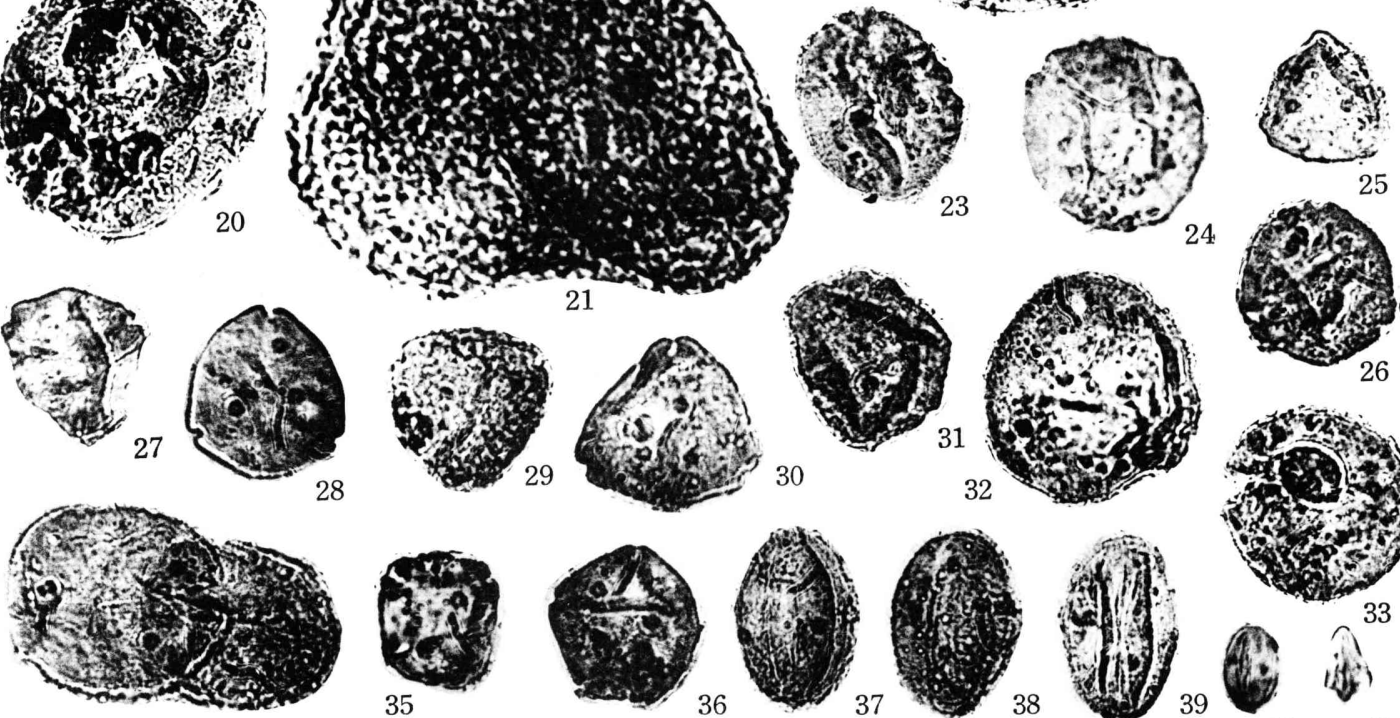

34
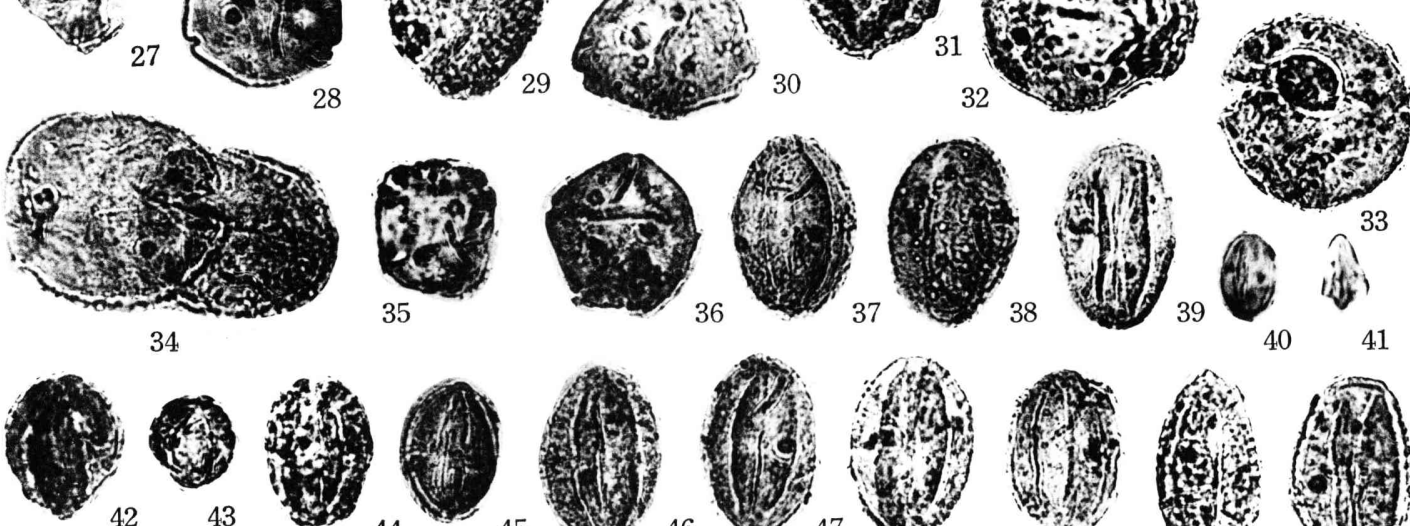
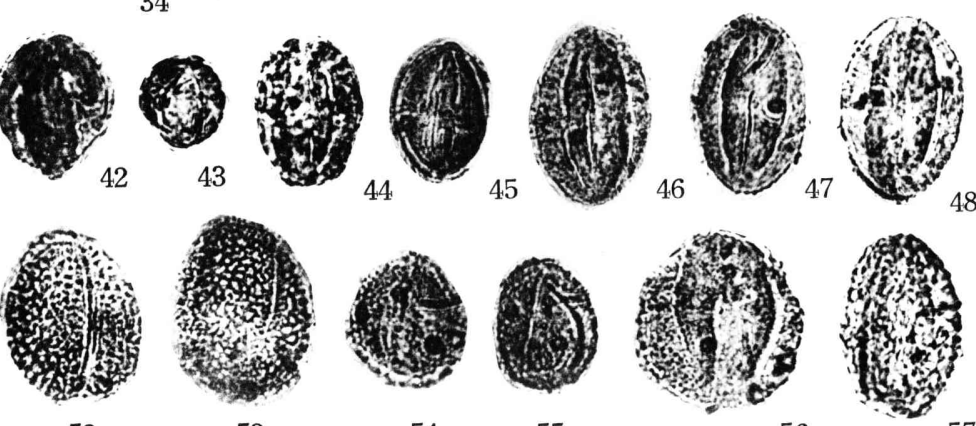

52

53

54

55

56

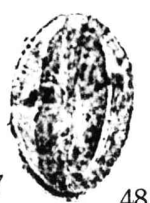

,
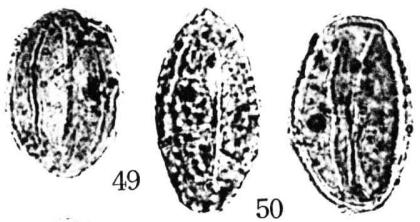

K. Takahashi: Palynologisch-stratigraphische Untersuchung. 


\section{Erklärung der Tafel 39}

Kasuya (Fig. 1-8) und Fukuoka Kohlenfeld (Fig. 9-63)

(Objekte ausser Fig. 19 etwa $600 \times$ )

Fig. 1. Tricolporopoll. asperatus $\mathrm{T}_{\Delta \mathrm{K}}$. (MS) Funaishi Fz. (Shin-otani Kbw.)

Fig. 2-3. Tricolporopoll. excellens $\mathrm{T}_{\mathrm{AK}}$. (MS) Urata Fz. (Fukuhaku Kbw.)

Fig. 4-7. Tricolporopoll. claviger $\mathrm{T}_{\mathrm{AK}}$. (MS) Urata Fz. (Fukuhaku Kbw.)

Fig. 8. Tricolporopoll. tertiarius $\mathrm{T}_{\mathrm{AK}}$ (MS) Urata Fz. (Fukuhaku Kbw.)

Fig. 9. Stereispor.? sp. Futae Fz. (Tashima Kbw).

Fig. 10. Laevigatospor. dehiscens $\mathrm{T}_{\mathrm{AK}}$. (MS) Yonshaku Fz. (Sawara Kbw.)

Fig. 11. Monocolpopoll. universalis $\mathrm{T}_{\mathrm{AK}}$ n. sp. Futae Fz. (Tashima Kbw.)

Fig. 12. Monocolpopoll. sp. Yonshaku Fz. (Sawara Kbw.)

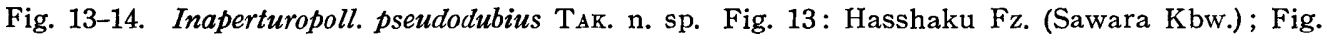
14: Goshaku Fz. (Fukutoyo Kbw.)

Fig. 15. Inaperturopoll. ligularis $\mathrm{T}_{\mathrm{AK}}$. (MS) Yonshaku Fz. (Sawara Kbw.)

Fig. 16. Inaperturopoll. laevigatus TAK. n. sp. (Typus); Yonshaku Fz. (Sawara Kbw.)

Fig. 17-18. Inaperturopoll. crassatus $\mathrm{T}_{\mathrm{AK}}$. (MS) Yonshaku Fz. (Sawara Kbw.)

Fig. 19. Pityospor. sp. etwa $300 \times$; Hitoe Fz. (Tashima Kbw.)

Fig. 20-21. Triporopoll. shimensis Tak. (MS) Fig. 20: Hitoe Fz. (Tashima Kbw.) ; Fig. 21 : Hasshaku Fz. (Sawara Kbw.)

Fig. 22-23. Triporopoll. constatus TAK. (MS) Fig. 22: Goshaku Fz. (Fukutoyo Kbw.); Fig. 23: Futae Fz. (Tashima Kbw.)

Fig. 24-25. Triporopoll. festatus TAK. (MS) Fig. 24 : Nanashaku-Nishaku Fz. (Sawara Kbw.); Fig. 25: Futae Fz. (Tashima Kbw.)

Fig. 26. Subtriporopoll. kyushuensis $\mathrm{T}_{\mathrm{AK}}$. (MS) Obitan Fz. (Sawara Kbw.)

Fig. 27-29. Polyvestibulopoll. eminens $\mathrm{T}_{\mathrm{AK}}$. (MS) Futae Fz. (Tashima Kbw.)

Fig. 30. Polyporopoll grandis TAK. (MS) Futae Fz. (Tashima Kbw.)

Fig. 31-32. Tricolpopoll. umiensis $\mathrm{T}_{A \mathrm{~K}}$. n. sp. Fig. 31 : Nanashaku-Nishaku Fz. (Sawara Kbw.); Fig. 32 : Futae Fz. (Tashima Kbw.)

Fig. 33-34. Tricolpopoll. sculptus $\mathrm{T}_{\mathrm{AK}}$. n. sp. Hitoe Fz. (Tashima Kbw.)

Fig. 35-37. Tricolpopoll. liblarensis (Тномs.) subsp. fallax (R. Рот.) Fig. 35, 36: Goshaku Fz. (Fukutoyo Kbw.); Fig. 37 : Hasshaku Fz. (Sawara Kbw.)

Fig. 38. Tricolpopoll. vulgaris $\mathrm{T}_{A \mathrm{~K}}$. n. sp. Nanashaku-Nishaku Fz. (Sawara Kbw.)

Fig. 39-44. Tricolpopoll. ditis TAK. n. sp. Fig. 39, 41, 44: Nanashaku-Nishaku Fz. (Sawara Kbw.); Fig. 40, 42,43 : Hasshaku Fz. (Sawara Kbw.)

Fig. 45-47. Tricolpopoll. meinohamensis $\mathrm{T}_{\mathrm{AK}}$. (MS) Yonshaku Fz. (Sawara Kbw.)

Fig. 48. Tricolpopoll. facetus $\mathrm{T}_{\mathrm{AK}}$. (MS) Hasshaku Fz. (Sawara Kbw.)

Fig. 49. Tricolpopoll inamoenus TAK. (MS) Goshaku Fz. (Fukutoyo Kbw.)

Fig. 50-51. Tricolpopoll. rudis $\mathrm{T}_{\mathrm{AK}}$. (MS) Futae Fz. (Tashima Kbw.)

Fig. 52. Tricolpopoll. reticulatus TAK. (MS) Futae Fz. (Tashima Kbw.)

Fig. 53, 60. Tricolpopoll. microreticulatus $\mathrm{T}_{\mathrm{AK}}$. (MS) Fig. 53: Futae Fz. (Tashima Kbw.); Fig. 60 : Nanashaku-Nishaku Fz. (Sawara Kbw.)

Fig. 54-56. Tricolporopoll. sp. Fig. 54: Nanashaku Fz. (Sawara Kbw.) Fig. 55, 56: Hasshaku Fz. (Sawara Kbw.)

Fig. 57. Tricolporopoll. asperatus $\mathrm{T}_{\mathrm{AK}}$ (MS) Hasshaku Fz. (Sawara Kbw.)

Fig. 58-59a. Tricolporopoll. incertus TAK. (MS) Hasshaku Fz. (Sawara Kbw.)

Fig. 59b. Tricolpopoll. liblarensis (Tномs.) subsp. fallax (R. Poт.) Hasshaku Fz. (Sawara Kbw.)

Fig. 61. Tricolporopoll. excellens $\mathrm{T}_{\mathrm{AK}}$. (MS) Futae Fz. (Tashima Kbw.)

Fig. 62. Tricolporopoll claviger $\mathrm{T}_{\mathrm{AK}}$. (MS) Futae Fz. (Tashima Kbw.)

Fig. 63. Tricolporopoll. tertiarius $\mathrm{T}_{\mathrm{AK}}$. (MS) Futae Fz. (Tashima Kbw.)

Fig. 64-66. Pilzreste 

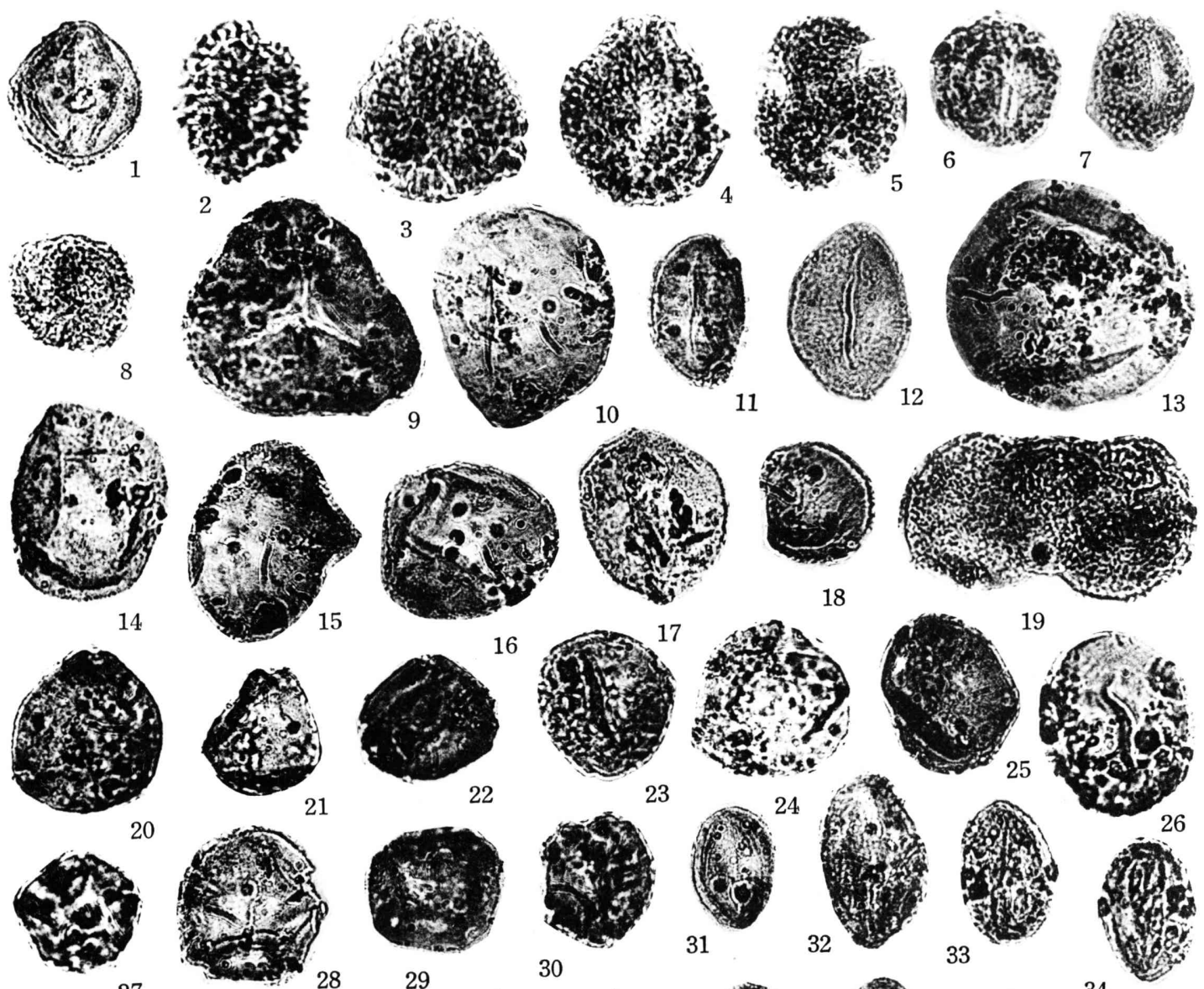

解
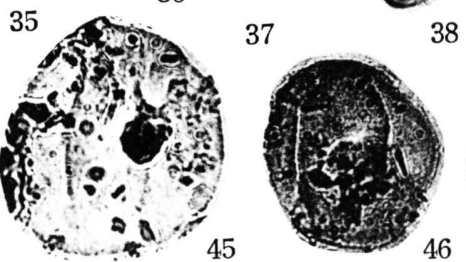

38
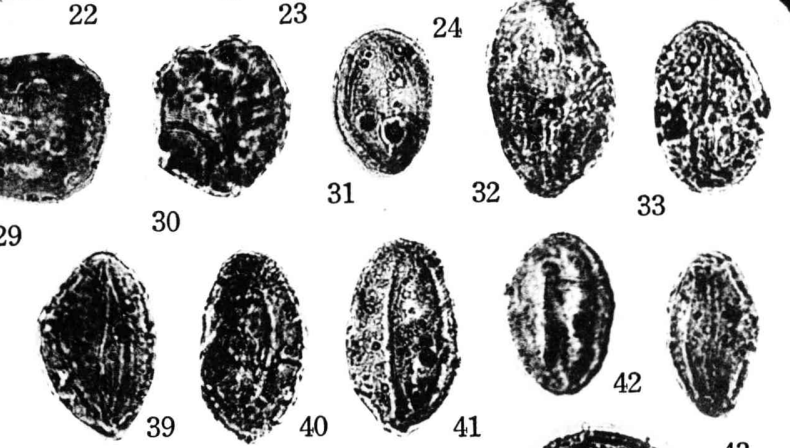

34
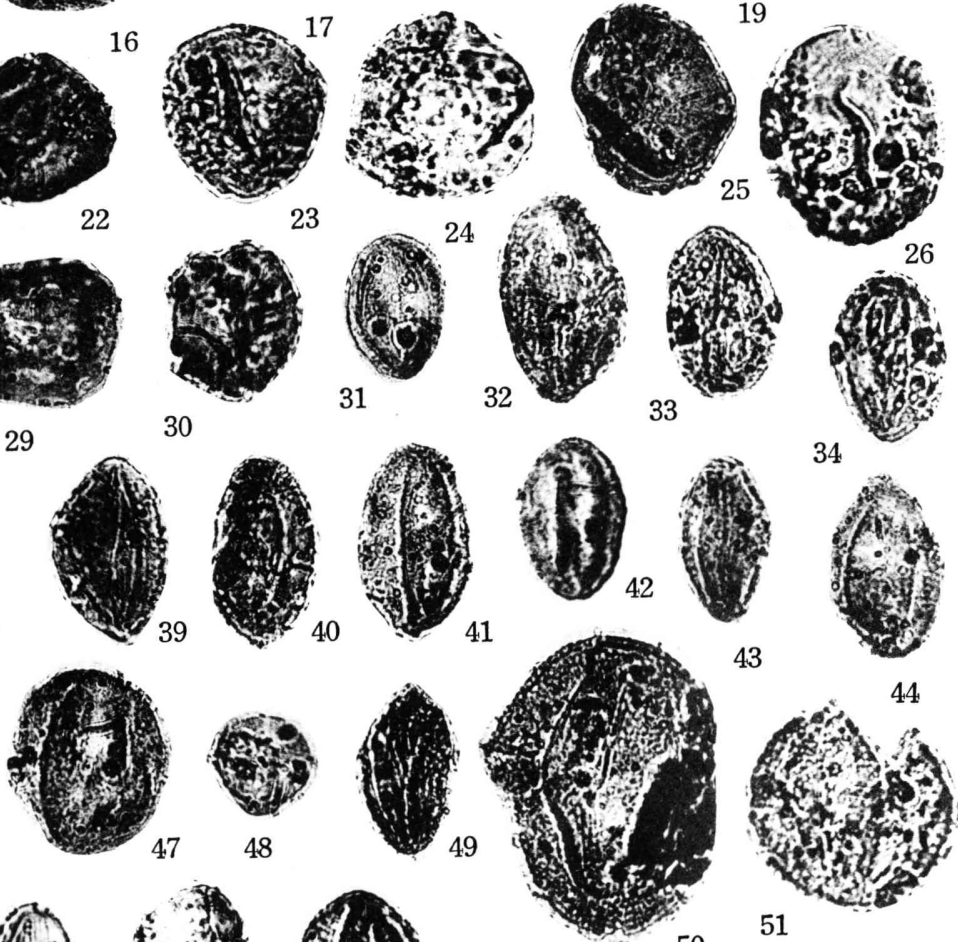

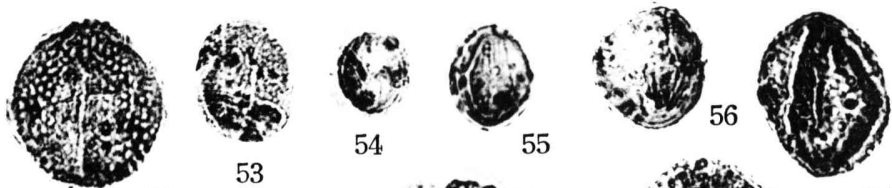

52

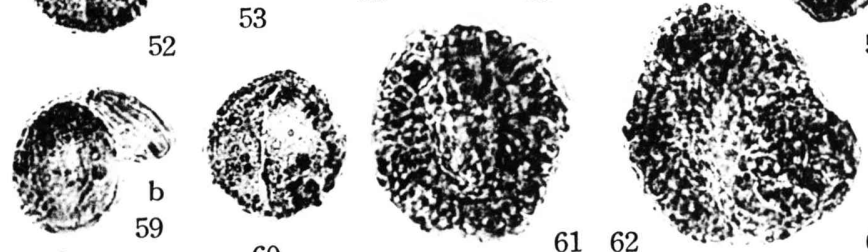

60
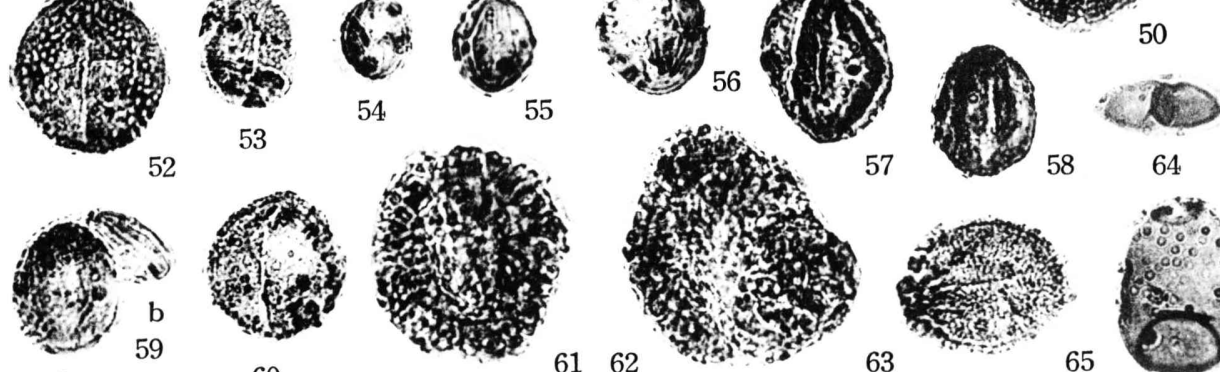

a

60
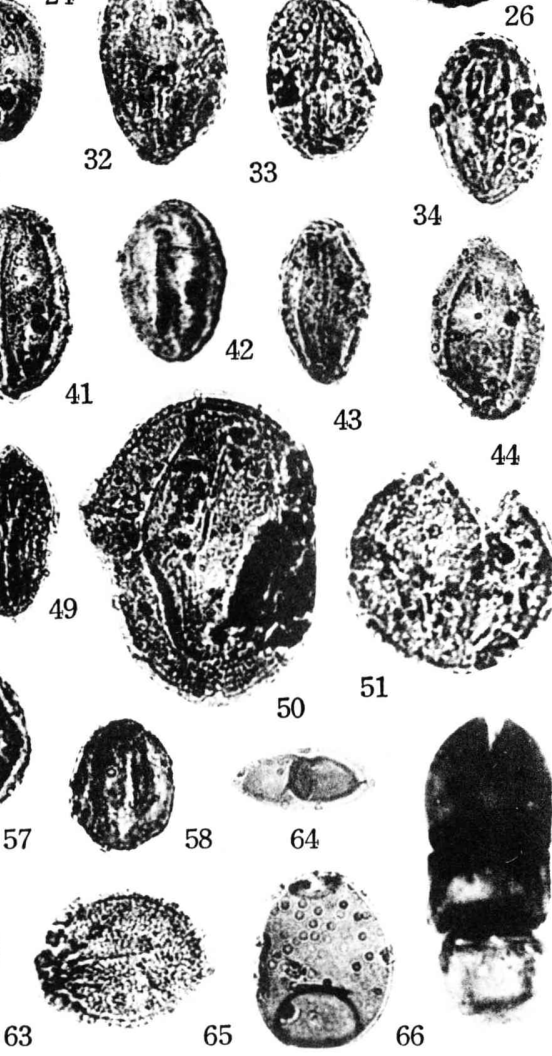

K. TAkahashi : Palynologisch-stratigraphische Untersuchung. 